\title{
A relaxation result for micromagnetics in SBV
}

\author{
Pedro M. Santos
}

Abstract. An integral representation for the functional

$$
\begin{aligned}
\mathcal{F}(m, M):=\inf \left\{\liminf _{k \rightarrow+\infty} \int_{\Omega} f\left(x, m_{k}(x), \nabla m_{k}(x)\right) d x+\int_{\Omega \cap S\left(m_{k}\right)}\left|\left[m_{k}\right](x)\right| d \mathcal{H}^{N-1}:\right. \\
m_{k} \in S B V\left(\Omega ; \mathbb{R}^{N}\right), \quad\left|m_{k}(x)\right|=1 \text { a.e. in } \Omega, \\
\left.m_{k} \rightarrow m \text { in } L^{1}\left(\Omega ; \mathbb{R}^{N}\right), \quad \nabla m_{k} \rightarrow M \text { in } L^{2}\left(\Omega ; \mathbb{R}^{N}\right)\right\}
\end{aligned}
$$

is obtained. This problem is motivated by equilibria issues in micromagnetics.

Mathematics Subject Classification (2000). 35D99, 35E99, 49J45.

Keywords. Integral representation, Quasiconvexification, Relaxation, SBV-functions.

\section{Introduction}

In this paper we consider the functional

$$
E(m)=\int_{\Omega} f(x, m(x), \nabla m(x)) d x+\int_{\Omega \cap S(m)}|[m](x)| d \mathcal{H}^{N-1}+E_{1}(m),
$$

where $\Omega \subset \mathbb{R}^{N}$ is an open bounded set, $m \in S B V\left(\Omega ; \mathbb{R}^{N}\right)$ is subject to the pointwise constraint $|m(x)|=1$ a.e. in $\Omega$, and $E_{1}$ is a continuous functional with respect to the strong topology of $L^{2}$.

The motivation to address this type of energies is drawn from micromagnet$i c s$, a continuum model to describe the behavior of a ferromagnetic body. According to this theory, the equilibrium states of a body subject to a given external field $h_{e}$ correspond to absolute (or local) minimizers of the energy functional 


$$
\begin{aligned}
m & \rightarrow \alpha \int_{\Omega}|\nabla m(x)|^{2}+\int_{\Omega} \varphi(m(x)) d x-\int_{\Omega} h_{e}(x) \cdot m(x) d x \\
& +\frac{1}{2} \int_{\mathbb{R}^{3}}\left|h_{m}(x)\right|^{2} d x,
\end{aligned}
$$

where $\Omega \subset \mathbb{R}^{3}$ represents a region occupied by the body and the magnetization is a function $m: \mathbb{R}^{3} \rightarrow \mathbb{R}^{3}$ such that

$$
|m(x)|=m_{s} \chi_{\Omega}(x) \text { for } \mathcal{L}^{3} \text { a.e. } x \in \mathbb{R}^{3},
$$

and $m_{s}>0$, the saturation magnetization, is a function of the temperature and of the specific material. The induced magnetic field is a function $h: \mathbb{R}^{3} \rightarrow \mathbb{R}^{3}$ which is related to $m$ through (distributional) Maxwell's equations, i.e.

$$
\left\{\begin{array}{l}
\operatorname{curl} h_{m}=0 \text { in } \mathbb{R}^{3}, \\
\operatorname{div}\left(m+h_{m}\right)=0 \text { in } \mathbb{R}^{3} .
\end{array}\right.
$$

We note that the last two integrals in (1.2) are continuous with respect to the strong topology in $L^{2}$ (see $[12,15]$ ), and their sum reduces to the term $E_{1}$. By considering a surface term in (1.1) we allow the possibilities of $m$ to have discontinuities (magnetic cracks), the body be made of several magnetic materials, or both (see [1] for some arguments concerning the penalization of formation of interfaces).

When the functional (1.1) is not lower semicontinuous, it is usual to look for its relaxation, i.e., given a magnetization $m$ we want to attain it by spending the least possible energy, and this corresponds to characterizing the functional below

$$
\begin{gathered}
F(m):=\inf \left\{\liminf _{k \rightarrow+\infty} E\left[m_{k}\right]: m_{k} \in S B V\left(\Omega ; \mathbb{R}^{N}\right), \quad\left|m_{k}(x)\right|=1 \text { a.e. in } \Omega,\right. \\
\left.m_{k} \rightarrow m \text { in } L^{1}\left(\Omega ; \mathbb{R}^{N}\right)\right\} .
\end{gathered}
$$

We consider here a surface term that will induce interaction, i.e., for sequences $\left\{m_{k}\right\}$ with bounded energy we may not have $\nabla m_{k} \rightarrow \nabla m$ and $D^{s} m_{k} \rightarrow D^{s} m$, instead it may happen that part of $\nabla m$ is approached in a more economical way using jumps. Adopting a point of view similar to Choksi and Fonseca [8] we consider a relaxed energy which also take in acount the limits of the gradients

$$
\begin{array}{r}
\mathcal{E}(m, M):=\inf \left\{\liminf _{k \rightarrow+\infty} E\left[m_{k}\right]: m_{k} \in S B V\left(\Omega ; \mathbb{R}^{N}\right), \quad\left|m_{k}(x)\right|=1 \text { a.e. in } \Omega,\right. \\
\left.m_{k} \rightarrow m \quad \text { in } \quad L^{1}\left(\Omega ; \mathbb{R}^{N}\right), \quad \nabla m_{k} \rightarrow M \quad \text { in } L^{2}\left(\Omega ; \mathbb{R}^{N}\right)\right\} .
\end{array}
$$

Similar relaxed energies were considered in [8], where they studied relaxed energies associated with structured deformations of continua, a concept introduced by Del Piero and Owen [13] for taking into account situations where the deformation of a body can be attained via a diffusion of cracks (microscopic disarrangements). 
In [13] they treat a triples $(K, g, G)$, where $K$ is the macroscopic crack, $g$ is the macroscopic deformation, $G$ is the deformation without disarrangements, and the regions where there is presence of microscopic disarrangements are identified with $\{\nabla g \neq G\}$. In [8] these triplets are reduced to pairs $(g, G)$, and the set $K$ is incorporated in $g$ by identifying crack sites with jump sets of $S B V$ functions.

Here we make the parallel of what was done in [8] to the case of micromagnetics. The minimum energy can be attained by sequences such that there is a diffusion of discontinuities in some region (microscopic disarrangements), which in the limit can be identified by considering pairs $(m, M)$ and through the inequality $\nabla m \neq M$. Indeed, if $\nabla m \neq M$ in some open set $A$ and if we have a sequence of magnetizations $\left\{m_{k}\right\} \subset L^{1}\left(\Omega ; S^{N-1}\right)$, with $m_{k} \rightarrow m$ in $L^{1}$, $\nabla m_{k} \rightarrow M$ in $L^{2}$, we know by the compactness theorem of Ambrosio [3,6] that necessarily $\mathcal{H}^{N-1}\left(A \cap S\left(m_{k}\right)\right) \rightarrow+\infty$, i.e., there is a diffusion of discontinuities through $A$. A pair $(m, M)$ gives a more complete description of the minimizers, because we not only know what is the magnetization, but also we get information about the microscopic disarrangements.

We note that the natural space for the relaxed energy would be with $m \in$ $B V$, but due to the superlinear growth of the bulk density sequences with nontrivial Cantor part in the limit have infinite energy.

The function $f: \Omega \times \mathbb{R}^{N} \times \mathbb{R}^{N^{2}} \rightarrow[0,+\infty)$ is assumed to be Caratheódory and satisfies the growth condition

$$
\frac{1}{C}|v|^{2}-C \leq f(x, y, v) \leq C\left(1+|v|^{2}\right)
$$

for some $C>0$. In addition, the following hold:

for every $\left(x_{0}, u_{0}\right) \in \Omega \times \mathbb{R}^{N}$ and $\varepsilon>0$ there exists $\delta>0$ such that

$$
f\left(x_{0}, u_{0}, v\right)-f(x, u, v) \leq \varepsilon(1+f(x, u, v))
$$

for all $(x, u) \in \Omega \times \mathbb{R}^{N}$ with $\left|x-x_{0}\right|+\left|u-u_{0}\right|<\delta$ and all $v \in \mathbb{R}^{N \times N}$, for every compact $K \subset \Omega \times \mathbb{R}^{N}$ exits $L_{K}>0$ such that

$$
\left|f(x, u, v)-f\left(x, u, v^{\prime}\right)\right| \leq L_{K}\left(1+|v|+\left|v^{\prime}\right|\right)\left|v-v^{\prime}\right|
$$

for all $(x, u) \in K$ and $v \in \mathbb{R}^{N \times N}$.

Since $m_{k} \rightarrow m$ in $L^{2}\left(\Omega ; \mathbb{R}^{N}\right)$ (what follows immediately from the $L^{1}$ convergence and the bounds) implies $E_{1}\left(m_{k}\right) \rightarrow E(m)$, we have that

$$
\mathcal{E}(m, M)=\mathcal{F}(m, M)+E_{1}(m),
$$


where $\mathcal{F}$ is given by

$$
\begin{aligned}
\mathcal{F}(m, M):=\inf \left\{\liminf _{k \rightarrow+\infty} \int_{\Omega} f\left(x, m_{k}(x), \nabla m_{k}(x)\right) d x+\int_{\Omega \cap S\left(m_{k}\right)}\left|\left[m_{k}\right](x)\right| d \mathcal{H}^{N-1}:\right. \\
m_{k} \in S B V\left(\Omega ; \mathbb{R}^{N}\right), \quad\left|m_{k}(x)\right|=1 \text { a.e. in } \Omega \\
\left.m_{k} \rightarrow m \quad \text { in } L^{1}\left(\Omega ; \mathbb{R}^{N}\right), \quad \nabla m_{k} \rightarrow M \quad \text { in } L^{2}\left(\Omega ; \mathbb{R}^{N}\right)\right\}
\end{aligned}
$$

Since strong convergence in $m$ entails the convergence of the nonlocal term $\int_{\Omega}\left|h_{m}\right|^{2} d x$, the relaxation will not involve directly Maxwell's equations. Relaxation results in the context of micromagnetics were also studied by Fonseca and Leoni [14], where they consider the non-exchange model (without the first term in (1.2), i.e. the exchange energy, see [12]) and they find the relaxation of the functional

$$
G(m):=\int_{\mathbb{R}^{N}} g\left(x, \chi_{\Omega}(x) m(x), u(x), \nabla u(x)\right) d x,
$$

with respect to $L^{\infty}$-weak ${ }^{*}$ convergence for $m$, where $\left(\chi_{\Omega} m, \nabla u\right)$ satisfies the Maxwell's equations, i.e. $u \in H^{1}\left(\mathbb{R}^{N}\right)$ is the unique solution of $\triangle u+\operatorname{div}\left(\chi_{\Omega} m\right)=0$ in $\mathbb{R}^{N}$. In [14] they obtained a representation formula involving a quasiconvexification of $g$ which takes into account the underlying partial differential equation.

The main result of this paper is

Theorem 1.1. The functional $\mathcal{F}$ has an integral representation of the form

$$
\mathcal{F}(m, M)=\int_{\Omega} H(x, m(x), \nabla m(x), M(x)) d x+\int_{\Omega \cap S(m)}|[m](x)| \mathcal{H}^{N-1},
$$

where for $x_{0} \in \Omega, a \neq 0, A, B \in \mathbb{R}^{N \times N}$,

$$
\begin{gathered}
H\left(x_{0}, a, A, B\right):=\inf \left\{\int_{Q} \bar{f}\left(x_{0}, a, \nabla u(x)\right) d x+\int_{Q \cap S(u)}|[u](x)| d \mathcal{H}^{N-1}(x):\right. \\
u \in S B V\left(Q ; \mathbb{R}^{N}\right) \cap L^{\infty}\left(Q ; \mathbb{R}^{N}\right),\left.\quad u\right|_{\partial Q}=A x, \\
\left.\nabla u \in L^{2}, \quad \int_{Q} \nabla u=B\right\},
\end{gathered}
$$

$\bar{f}\left(x_{0}, a, v\right):=f\left(x_{0}, a, P_{a}(v)\right)$ and $P_{a}: \mathbb{R}^{N} \rightarrow \mathbb{R}^{N}$ is the orthogonal projection of $\mathbb{R}^{N}$ onto $T_{a}\left(S^{N-1}\right)$ (the tangent space to $|a| S^{N-1}$ at the point a).

The problem of relaxing a functional under a manifold constraint was already treated by Dacorogna et al. [10], where they obtained the representation result

$$
\begin{aligned}
\mathcal{F}(u):=\inf \left\{\liminf _{n \rightarrow+\infty} \int_{\Omega} f\left(\nabla u_{n}(x)\right) d x: u_{n} \rightarrow u \text { in } W^{1, p},\right. \\
u(x) \in \mathcal{M} \text { for a.e } x \in \Omega\}=\int_{\Omega} Q_{T} f(u(x), \nabla u(x)) d x,
\end{aligned}
$$


with $Q_{T}$ the tangential quasiconvexification defined by

$$
Q_{T}(a, v):=\inf \left\{\int_{Q} f(v+\nabla \varphi(x)) d x: \varphi \in W_{0}^{1, \infty}\left(Q ; T_{a}(\mathcal{M})\right\},\right.
$$

for $a \in \mathcal{M}, v \in T_{a}(\mathcal{M})^{N}$, and $\mathcal{M}$ is a $C^{1}$ manifold. As shown in [10], an alternative formula for $Q_{T}$ is

$$
Q_{T}(a, v)=Q \bar{f}(a, v),
$$

where $\bar{f}(a, v)=f\left(a, P_{a}(v)\right), P_{a}$ denotes the projection into $T_{a}(\mathcal{M})$ and $Q$ refers to the usual quasiconvexification $[9,16]$, i.e.

$$
Q \bar{f}(a, v):=\inf \left\{\int_{Q} \bar{f}(a, v+\nabla \varphi(x)) d x: \varphi \in W_{0}^{1, \infty}\left(Q ; \mathbb{R}^{d}\right)\right\} .
$$

Relaxation of functionals under constraints are also treated in [7].

\section{The spaces BV, SBV, and sets of finite perimeter}

We introduce some notation that we will be using throughout the paper. Let $\Omega$ be an open, bounded subset of $\mathbb{R}^{N}$. Let $\nu \in S^{N-1}$, and denote by $Q_{\nu}$ the unit cube centered at the the origin and with two faces normal to $\nu$, i.e.,

$$
Q_{\nu}:=\left\{x \in \mathbb{R}^{N}:\left|x . \nu_{i}\right|<\frac{1}{2}, \quad|x . \nu|<\frac{1}{2}, i=1 \ldots N-1\right\}
$$

for some orthonormal basis $\left\{\nu_{1}, \ldots, \nu_{N-1}, \nu\right\}$ of $\mathbb{R}^{N}$; we abbreviate $Q_{e_{N}}$ as $Q$ and we write $Q_{\nu}(a, r):=a+r Q_{\nu}, a \in \mathbb{R}^{N}, r>0$. Let $\Pi(x):=\frac{x}{|x|}$.

We recall some basic definitions and properties of the space BV of functions of bounded variation, of the space SBV of functions of special bounded variation, and also of sets of finite perimeter. For more details we refer the reader to [6].

Definition 2.1. A function $u \in L^{1}\left(\Omega ; \mathbb{R}^{d}\right)$ is said to be of bounded variation, $u \in$ $B V\left(\Omega ; \mathbb{R}^{d}\right)$, if for all $i \in\{1, \ldots, d\}, j \in 1, \ldots, N$, there exists a bounded Radon measure $\mu_{i j}$ such that

$$
\int_{\Omega} u_{i}(x) \frac{\partial \varphi}{\partial x_{j}}(x) d x=-\int_{\Omega} \varphi(x) d \mu_{i j}
$$

for every $\varphi \in C_{c}^{1}(\Omega)$. The distributional derivative $D u$ is the matrix-valued measure with components $\mu_{i j}$. The total variation of the gradient measure, $|D u|(\Omega)$, is given by $|D u|(\Omega)=\sum_{1}^{d}\left|D u_{i}\right|(\Omega)$, where

$$
\left|D u_{i}\right|(\Omega):=\sup _{\varphi}\left\{\int_{\Omega} u_{i} \operatorname{div} \varphi d x: \varphi \in C_{c}^{1}\left(\Omega ; \mathbb{R}^{N}\right), \quad\|\varphi\|_{\infty} \leq 1\right\} .
$$

The space $B V$ is a Banach space equipped with the norm

$$
\|u\|_{B V\left(\Omega ; \mathbb{R}^{d}\right)}:=\|u\|_{L^{1}\left(\Omega ; \mathbb{R}^{d}\right)}+|D u|(\Omega) .
$$


Definition 2.2. A set $A \subset \Omega$ is said to be of finite perimeter in $\Omega$ if $\chi_{A} \in B V(\Omega)$, where $\chi_{A}$ denotes the characteristic function of $A$. The perimeter of $A$ is defined by

$$
\operatorname{Per}_{\Omega}(A):=\left|D \chi_{A}\right|(\Omega)
$$

There is an important connection between sets of finite perimeter and level sets of $B V$ functions which we state next.

Theorem 2.3. (Co-area formula for $B V$ functions) If $u \in B V(\Omega)$, then $E_{t}:=\{x \in$ $\Omega: u(x)>t\}$ has finite perimeter for a.e. $t \in \mathbb{R}$, and

$$
|D u|(\Omega)=\int_{-\infty}^{+\infty}\left|D \chi_{E_{t}}\right|(\Omega) d t
$$

Let $E \subset \mathbb{R}^{N}$ be a $\mathcal{L}^{N}$-measurable set. We define

$$
E^{1}:=\left\{x \in \mathbb{R}^{N}: \lim _{\varepsilon \rightarrow 0} \frac{\mathcal{L}^{N}(E \cap Q(x, \varepsilon))}{\varepsilon^{N}}=1\right\}
$$

and

$$
E^{0}:=\left\{x \in \mathbb{R}^{N}: \lim _{\varepsilon \rightarrow 0} \frac{\mathcal{L}^{N}(E \cap Q(x, \varepsilon))}{\varepsilon^{N}}=0\right\},
$$

the measure theoretic interior (the set of points of density 1 in $E$ ) and the measure theoretic exterior (the set of points of density 0 in $E$ ) of $E$, respectively. Also, $\partial^{*} E$ is the essential boundary of $E$, i.e.

$$
\partial^{*} E:=\mathbb{R}^{N} \backslash\left(E^{0} \cup E^{1}\right) .
$$

We note that $\mathcal{L}^{N}\left(E \triangle E^{1}\right)=0$ and $\mathcal{L}^{N}\left(\left(\mathbb{R}^{N} \backslash E\right) \triangle E^{0}\right)=0$.

For sets of finite perimeter it is possible to define a normal vector on part of the boundary, the reduced boundary $\mathcal{F} E$.

Definition 2.4. Let $E \subset \Omega$ be a set of finite perimeter in $\Omega$. We define reduced boundary $\mathcal{F} E$ to be the set of points $x$ such that

(i) $\left|D \chi_{E}\right|(Q(x, \varepsilon))>0$ for every $\varepsilon>0$ such that $Q(x, \varepsilon) \subset \Omega$,

(ii) $\nu_{E}(x):=\lim _{\varepsilon \rightarrow 0} \frac{D \chi_{E}(Q(x, \varepsilon))}{\left|D \chi_{E}\right|(Q(x, \varepsilon)}$ exists in $\mathbb{R}^{N}$,

(iii) $\left|\nu_{E}(x)\right|=1$.

The function $\nu_{E}: \mathcal{F} E \rightarrow S^{N-1}$ is called the generalized inner normal to E.

It can be shown that $\mathcal{F} E \subset \partial^{*} E$ and $\mathcal{H}^{N-1}\left(\partial^{*} E \backslash \mathcal{F} E\right)=0$ (see [6]). The set $\mathcal{F} E$ is $(N-1)$-rectifiable, i.e.,

$$
\mathcal{F} E=\bigcup_{n=1}^{\infty} K_{n} \cup E
$$

and $\mathcal{H}^{N-1}(E)=0, K_{n}$ is a compact subset of a $C^{1}$ hypersurface $S_{n}$ for each $n$, and $\nu_{E} \mid S_{n}$ is normal to $S_{n}$. 
Given $u \in B V\left(\Omega ; \mathbb{R}^{d}\right)$, the approximate upper and lower limit of each component $u_{i}, i \in\{1, \ldots, d\}$, are given by

$$
\begin{gathered}
u_{i}^{+}(x):=\inf \left\{t \in \mathbb{R}: \lim _{\varepsilon \rightarrow 0^{+}} \frac{\mathcal{L}^{N}\left(\left\{u_{i}>t\right\} \cap Q(x, \varepsilon)\right)}{\varepsilon^{N}}=0\right\} \\
u_{i}^{-}(x):=\sup \left\{t \in \mathbb{R}: \lim _{\varepsilon \rightarrow 0^{+}} \frac{\mathcal{L}^{N}\left(\left\{u_{i}<t\right\} \cap Q(x, \varepsilon)\right)}{\varepsilon^{N}}=0\right\} .
\end{gathered}
$$

The set

$$
S(u):=\bigcup_{i=1}^{d}\left\{x \in \Omega: u_{i}^{-}(x)<u_{i}^{+}(x)\right\}
$$

is called jump set of $u$, and the value $\tilde{u}(x):=\frac{1}{2}\left(u^{+}(x)+u^{-}(x)\right)$ is defined for every $x \in \Omega$. It is well known that $S(u)$ is $(N-1)$-rectifiable.

The result below is about some fine properties that $B V$ functions enjoy.

Theorem 2.5. If $u \in B V\left(\Omega ; \mathbb{R}^{d}\right)$, then

(i) for $\mathcal{L}^{N}$-a.e. $x \in \Omega$,

$$
\lim _{\varepsilon \rightarrow 0^{+}} \frac{1}{\varepsilon^{N+1}}\left\{\int_{Q\left(x_{0}, \varepsilon\right)}|u(y)-u(x)-\nabla u(x) \cdot(y-x)|^{\frac{N}{N-1}} d y\right\}^{\frac{N-1}{N}}=0
$$

(ii) for $\mathcal{H}^{N-1}$-a.e. $x \in S(u)$, there exists a unit vector $\nu(x) \in S^{N-1}$, normal to $S(u)$ at $x$, and there exist vectors $u_{-}(x), u_{+}(x) \in \mathbb{R}^{d}$ such that

$$
\begin{aligned}
& \lim _{\varepsilon \rightarrow 0^{+}} \frac{1}{\varepsilon^{N}} \int_{\left\{y \in Q_{\nu(x)}(x, \varepsilon):(y-x) \cdot \nu(x)>0\right\}}\left|u(y)-u_{+}(x)\right|^{\frac{N}{N-1}} d y=0 ; \\
& \lim _{\varepsilon \rightarrow 0^{+}} \frac{1}{\varepsilon^{N}} \int_{\left\{y \in Q_{\nu(x)}(x, \varepsilon):(y-x) \cdot \nu(x)<0\right\}}\left|u(y)-u_{-}(x)\right|^{\frac{N}{N-1}} d y=0 ;
\end{aligned}
$$

(iii) for $\mathcal{H}^{N-1}$-a.e. $x_{0} \in \Omega \backslash S(u)$

$$
\lim _{\varepsilon \rightarrow 0^{+}} \frac{1}{\varepsilon^{N}} \int_{Q}\left|u(y)-\tilde{u}\left(x_{0}\right)\right| d x=0 .
$$

We remark that, in general, $\left(u_{i}\right)^{ \pm} \neq\left(u_{ \pm}\right)_{i}$. We denote by $[u](x)$ the jump of $u$ at $x$, defined by

$$
[u](x):=u_{+}(x)-u_{-}(x) .
$$

If $u \in B V\left(\Omega ; \mathbb{R}^{d}\right)$, then the measure $D u$ may be represented as

$$
D u=\nabla u \mathcal{L}^{N}+\left(u_{+}-u_{-}\right) \otimes \nu \mathcal{H}^{N-1}\lfloor S(u)+C(u),
$$

where $\nabla u$ is the density of the absolutely continuous part of $D u$ with respect to $\mathcal{L}^{N}$, and $C(u)$ is the Cantor part. The three measures in (2.1) are mutually singular.

It is possible to define the trace of a function $u \in B V\left(\Omega ; \mathbb{R}^{d}\right)$ on the reduced boundary of a set $E$ of finite perimeter in $\Omega$ (see Theorem 3.77., pp. 171 of [6]). 
Theorem 2.6. Let $u \in B V\left(\Omega ; \mathbb{R}^{d}\right)$ and let $E \subset \Omega$ be a set of finite perimeter in $\Omega$. For $\mathcal{H}^{N-1}$-almost every $x \in \mathcal{F} E$ there exist $u_{+, \mathcal{F} E}(x), u_{-, \mathcal{F} E}(x)$ in $\mathbb{R}^{d}$ such that

$$
\lim _{\varepsilon \rightarrow 0} \frac{1}{\varepsilon^{N}} \int_{Q_{\nu_{E}}^{+}(x, \varepsilon)}\left|u(y)-u_{+, \mathcal{F} E}(x)\right| d y=0
$$

and

$$
\lim _{\varepsilon \rightarrow 0} \frac{1}{\varepsilon^{N}} \int_{Q_{\nu_{E}}^{-}(x, \varepsilon)}\left|u(y)-u_{-, \mathcal{F} E}(x)\right| d y=0
$$

Moreover $D u\left\lfloor\mathcal{F} E=\left(u_{+, \mathcal{F} E}-u_{-, \mathcal{F} E}\right) \otimes \nu_{E} \mathcal{H}^{N-1}\lfloor\mathcal{F} E\right.$.

Theorem 2.7. Let $u, v \in B V\left(\Omega ; \mathbb{R}^{d}\right) \cap L^{\infty}$ and let $E$ be a set of finite perimeter in $\Omega$. Then $w:=u \chi_{E}+v \chi_{\Omega \backslash E} \in B V\left(\Omega ; \mathbb{R}^{d}\right)$ and

$$
D w=D u\left\lfloor E^{1}+\left(u_{+, \mathcal{F} E}-v_{-, \mathcal{F} E}\right) \otimes \nu_{E} \mathcal{H}^{N-1}\left\lfloor(\mathcal{F} E)+D v\left\lfloor E^{0}\right.\right.\right.
$$

The following subspace of $B V$ was introduced and studied by De Giorgi and Ambrosio [11].

Definition 2.8. A function $u \in B V\left(\Omega ; \mathbb{R}^{d}\right)$ is said to be of special bounded variation if $C(u)=0$. We write $u \in S B V\left(\Omega ; \mathbb{R}^{d}\right)$.

The next theorem is a generalization of the Besicovitch Differentiation Theorem (see Ambrosio and Dal Maso [5], Proposition 2.2).

Theorem 2.9. If $\lambda$ and $\mu$ are Radon measures in $\Omega, \mu \geq 0$, then there exists a Borel set $E \subset \Omega$ such that $\mu(E)=0$, and for every $x \in \operatorname{supp} \mu \backslash E$

$$
\frac{d \lambda}{d \mu}(x):=\lim _{\varepsilon \rightarrow 0} \frac{\lambda(x+\varepsilon C)}{\mu(x+\varepsilon C)}
$$

exists and is finite whenever $C$ is a bounded, convex, open set containing the origin.

The following $S B V$ compactness theorem is due to Ambrosio [3].

Theorem 2.10. Let $\varphi:[0,+\infty) \rightarrow \mathbb{R}$ and $\theta:(0,+\infty) \rightarrow \mathbb{R}$ be nondecreasing lower semicontinuous functions satisfying

$$
\lim _{t \rightarrow \infty} \frac{\varphi(t)}{t}=\infty, \quad \lim _{t \rightarrow 0^{+}} \frac{\theta(t)}{t}=\infty .
$$

Let $\left\{u_{n}\right\}$ be a sequence of functions in $S B V\left(\Omega ; \mathbb{R}^{d}\right) \cap L^{\infty}\left(\Omega ; \mathbb{R}^{d}\right)$ such that $\sup _{n}\left\|u_{n}\right\|_{\infty}<\infty$ and

$$
\sup _{n}\left\{\int_{\Omega} \varphi\left(\left|\nabla u_{n}\right|\right) d x+\int_{\Omega \cap S\left(u_{n}\right)} \theta\left(\left[u_{n}\right]\right) \mathcal{H}^{N-1}\right\}<\infty .
$$

Then there exists a subsequence $\left\{u_{n_{i}}\right\}$ and a function $u \in S B V\left(\Omega ; \mathbb{R}^{d}\right)$ such that

$$
u_{n_{i}} \rightarrow u \text { in } L^{1}, \quad \nabla u_{n_{i}} \rightarrow \nabla u \text { in } L^{1} .
$$

The next theorem was obtained by Alberti [2]. 
Theorem 2.11. Let $f \in L^{1}\left(\Omega ; \mathbb{R}^{d \times N}\right)$. There exists $u \in S B V\left(\Omega ; \mathbb{R}^{d}\right)$ and a Borel function $g: \Omega \rightarrow \mathbb{R}^{d \times N}$ such that

$$
D u=f \mathcal{L}^{N}+g \mathcal{H}^{N-1}\left\lfloor S(u), \quad \int_{\Omega \cap S(u)}|g| d \mathcal{H}^{N-1} \leq C\|f\|_{L^{1}\left(\Omega ; \mathbb{R}^{d \times N}\right)},\right.
$$

where $C$ depends only on $N$.

The next lemma is a simple corollary of the co-area formula for $B V$ functions, and it is an improvement to the Lemma 2.9. in [8].

Lemma 2.12. Let $u \in B V\left(\Omega ; \mathbb{R}^{d}\right)$. There exist functions $u_{n} \in S B V$, with $\nabla u_{n}=0$, such that $u_{n}-u \rightarrow 0$ in $L^{\infty}$ and

$$
\lim _{n \rightarrow+\infty}\left|D u_{n}\right|(\Omega)=\lim _{n \rightarrow+\infty} \int_{\Omega \cap S\left(u_{n}\right)}\left|\left[u_{n}\right](x)\right| d \mathcal{H}^{N-1}(x)=|D u|(\Omega) .
$$

Proof. Without loss of generality we may assume that $d=1$. We also assume $u$ to be non-negative; the general case follows by considering the positive and negative parts of $u$. Let $E_{t}:=\{x \in \Omega: u(x)>t\}$ and define

$$
u_{n}(x):=\sum_{i=1}^{\infty} \frac{1}{n} \chi_{E_{\left(a_{i n}\right)}}(x)
$$

where, using Theorem 2.3., $a_{i n} \in\left(\frac{i-1}{n}, \frac{i}{n}\right)$ is such that $E_{a_{i n}}$ has finite perimeter and

$$
\frac{\left|D \chi_{E_{a_{i n}}}\right|(\Omega)}{n} \leq \int_{\frac{i-1}{n}}^{\frac{i}{n}}\left|D \chi_{E_{t}}\right|(\Omega) d t
$$

Clearly $u_{n}-u \rightarrow 0$ in $L^{\infty}(\Omega)$, and

$$
\left|D u_{n}\right|(\Omega) \leq \sum_{i=0}^{\infty} \frac{1}{n}\left|D \chi_{E_{a_{i n}}}\right|(\Omega) \leq \int_{0}^{\infty}\left|D \chi_{E_{t}}\right|(\Omega) d t=|D u|(\Omega) .
$$

This yelds

$$
\limsup \left|D u_{n}\right|(\Omega) \leq|D u|(\Omega)
$$

The converse inequality follows from the lower semicontinuity of the total variation. 


\section{Proof of Theorem 1.1}

As it is usual in relaxation theory, we start by localizing $\mathcal{F}$, precisely, for every open subset $A \subset \mathbb{R}^{N}$ we define

$$
\begin{aligned}
\mathcal{F}[(m, M) ; A]:=\inf \left\{\liminf _{k \rightarrow+\infty} \int_{A} f\left(x, m_{k}(x), \nabla m_{k}(x)\right) d x\right. \\
+\int_{A \cap S\left(m_{k}\right)}\left|\left[m_{k}\right](x)\right| d \mathcal{H}^{N-1}: m_{k} \in S B V\left(A ; \mathbb{R}^{N}\right), \\
\left|m_{k}(x)\right|=1 \text { a.e. in } A, \\
\\
\left.m_{k} \rightarrow m \text { in } L^{1}\left(A ; \mathbb{R}^{N}\right), \quad \nabla m_{k} \rightarrow M \text { in } L^{2}\left(A ; \mathbb{R}^{N}\right)\right\} .
\end{aligned}
$$

We note that there is a compatibility condition linking $m$ and $M$, precisely, from the condition that $\nabla m_{k}(x) \in T_{m_{k}(x)}$ for a.e. $x \in \Omega$, which can be expressed by $m_{k}(x)^{T} \nabla m_{k}(x)=0$ a.e., passing to the limit we get $m(x)^{T} M(x)=0$ a.e. in $\Omega$. In view of this remark, in what follows we say that $(m, M)$ is admissible pair if $m \in S B V\left(\Omega ; \mathbb{R}^{N}\right), M \in L^{2}\left(\Omega ; \mathbb{R}^{N^{2}}\right),|m(x)|=1$ for a.e. $x \in \Omega$, and $m(x)^{T} M(x)=0$ a.e. in $\Omega$.

The goal is now to prove that for every admissible pair, $(m, M) \in S B V$ $\left(\Omega ; \mathbb{R}^{N}\right) \times L^{2}\left(\Omega ; \mathbb{R}^{N^{2}}\right),|m(x)|=1$ a.e. in $\Omega, m(x)^{T} M(x)=0$ a.e. in $\Omega, \mathcal{F}[(m, M) ;$. is the restriction of a Radon measure to $\mathcal{O}(\Omega)$, the set of all open subsets contained in $\Omega$. Once this is established, the integral representation will follow from the Radon-Nikodym Theorem.

The lemma below provides an alternative characterization of the density $H$.

Lemma 3.1. Under conditions (H2), (H3) we have

$$
\begin{aligned}
& H\left(x_{0}, a, A, B\right)=\inf \left\{\liminf _{n \rightarrow+\infty} \int_{Q} \bar{f}\left(x_{0}, a, \nabla u_{n}(y)\right) d y+\int_{Q \cap S\left(u_{n}\right)}\left|\left[u_{n}\right](y)\right| d \mathcal{H}^{N-1}(y):\right. \\
& u_{n} \in S B V\left(Q ; \mathbb{R}^{N}\right) \cap L^{\infty}\left(Q ; \mathbb{R}^{N}\right), \quad u_{n} \rightarrow A x \text { in } L^{1}, \\
&\left.\nabla u_{n} \rightarrow B \quad \text { in } \quad L^{2}\right\},
\end{aligned}
$$

and

$$
\begin{gathered}
H\left(x_{0}, a, A, B\right)=\inf \left\{\int_{Q} f\left(x_{0}, a, \nabla u(x)\right) d x+\int_{Q \cap S(u)}|[u](x)| d \mathcal{H}^{N-1}:\right. \\
u \in S B V\left(Q ; T_{a}\left(S^{N-1}\right)\right) \cap L^{\infty},\left.\quad u\right|_{\partial Q}=A x, \\
\left.\nabla u \in L^{2}, \quad \int_{Q} \nabla u(x) d x=B\right\}
\end{gathered}
$$

Proof. The proof of (3.1) is similar to the proof of Proposition 3.1. in [8] 
We start by noticing that one inequality $(\leq)$ in $(3.2)$ is trivial. For the converse inequality, we fix $A, B, N \times N$ matrices with columns in $T_{a}\left(S^{N-1}\right)$, and we consider a test function $u \in S B V\left(Q ; \mathbb{R}^{N}\right) \cap L^{\infty}$ such that $\nabla u \in L^{2}, u \mid \partial Q=$ $A x, \int_{Q} u(x) d x=B$. Set $\hat{u}:=P_{a} u$, and notice that $\hat{u} \in S B V\left(Q ; T_{a}\left(S^{N-1}\right)\right) \cap$ $L^{\infty},\left.\hat{u}\right|_{\partial Q}=A x, \nabla \hat{u} \in L^{2}$ and $\int_{Q} \nabla \hat{u}(x) d x=B$. Moreover, using the fact that $|[\hat{u}](x)| \leq|[u](x)|$ and the definition of $\bar{f}$, we get

$$
\begin{array}{rl}
\int_{Q} & f\left(x_{0}, a, \nabla \hat{u}(x)\right) d x+\int_{Q \cap S(\hat{u})}|[\hat{u}](x)| \mathcal{H}^{N-1}(x) \\
& \leq \int_{Q} \bar{f}\left(x_{0}, a, \nabla u(x)\right) d x+\int_{Q \cap S(u)}|[u](x)| \mathcal{H}^{N-1}(x),
\end{array}
$$

which proves the other inequality.

We now prove that any admissible pair $(m, M)$, can be attained by an admissible sequence, and we also obtain an upper bound for the relaxed energy.

Lemma 3.2. Let $A \subset \Omega$ be open, and let $(m, M)$ be an admissible pair. Then there exists a sequence $\left\{m_{k}\right\} \subset S B V\left(A ; \mathbb{R}^{N}\right),\left|m_{k}(x)\right|=1$ a.e. in $A$, such that

$$
m_{k} \rightarrow m \text { in } L^{1}\left(A ; \mathbb{R}^{N}\right), \quad \nabla m_{k} \rightarrow M \text { in } L^{2}\left(A ; \mathbb{R}^{N^{2}}\right) .
$$

Moreover,

$$
\mathcal{F}[(m, M) ; A] \leq C \int_{A}\left(1+|\nabla m(x)|^{2}+|M(x)|^{2}\right) d x+\int_{A \cap S(m)}|[m](x)| \mathcal{H}^{N-1}(x) .
$$

Proof. Using Theorem 2.11 we can find a function $h \in S B V\left(A ; \mathbb{R}^{N}\right)$ such that

$$
\nabla h=M-\nabla m, \quad|D h|(A) \leq C \int_{A}|M(x)-\nabla m(x)| d x .
$$

By Lemma 2.12 there exists a sequence $\left\{h_{k}\right\} \subset S B V\left(A ; \mathbb{R}^{N}\right), \nabla h_{k}=0$, such that

$$
h_{k}-h \rightarrow 0 \text { in } L^{\infty}\left(A ; \mathbb{R}^{N}\right), \quad\left|D h_{k}\right|(A) \rightarrow|D h|(A) .
$$

We consider

$$
m_{k}:=\Pi\left(m+h-h_{k}\right)
$$

where $\Pi(x)=x /|x|$ is a projection on the $N-1$-dimensional unit sphere. For $k$ large enough the sequence $m_{k}$ is well defined and belongs to $S B V\left(A ; \mathbb{R}^{N}\right)$, moreover it satisfies the constraint $\left|m_{k}(x)\right|=1$ a.e. in $A$. It is easy to check, taking into account that $\nabla \Pi(m(x)) \cdot M(x)=M(x)$ for a.e. $x \in A$, that $m_{k} \rightarrow m$ in $L^{1}\left(A ; \mathbb{R}^{N}\right)$ and $\nabla m_{k} \rightarrow M$ in $L^{2}\left(A ; \mathbb{R}^{N}\right)$. Given $\delta>0$, restrict $\Pi$ to a neighborhood of $S^{N-1}$ of the form $N_{\eta}:=\left\{x \in \mathbb{R}^{N}: 1-\eta<|x|<1+\eta\right\}$, with $\eta$ small enough, so that 
the Lipschitz constant of $\left.\Pi\right|_{N_{\eta}}$ will be smaller than $1+\delta$. Then we have, for $k$ large enough,

$$
\begin{aligned}
\mathcal{F}[(m, M) ; A] \leq & \liminf _{k \rightarrow+\infty}\left\{\int_{A} f\left(x, m_{k}(x), \nabla m_{k}(x)\right) d x+\int_{A \cap S\left(m_{k}\right)}\left|\left[m_{k}\right](x)\right| d \mathcal{H}^{N-1}\right\} \\
\leq & \limsup _{k \rightarrow+\infty}\left\{\int_{A} f\left(x, m_{k}(x), \nabla m_{k}(x)\right) d x+(1+\delta) \int_{A \cap S(m)}|[m](x)| \mathcal{H}^{N-1}(x)\right. \\
& \left.+(1+\delta)\left(\int_{A \cap S(h)}|[h](x)| \mathcal{H}^{N-1}(x)+\int_{A \cap S\left(h_{k}\right)}\left|\left[h_{k}\right](x)\right| \mathcal{H}^{N-1}(x)\right)\right\}
\end{aligned}
$$

and using $(H 1),(3.4)$ and (3.5), and the fact that $\delta$ is arbitrary we get the upper bound (3.3).

Now we prove a subadditivity property for $\mathcal{F}[(m, M) ;$.

Lemma 3.3. Let $A, B, C$ be open subsets of $\Omega$ such that $C \subset \subset B \subset \subset A$. Then

$$
\mathcal{F}[(m, M) ; A] \leq \mathcal{F}[(m, M) ; B]+\mathcal{F}[(m, M) ; A \backslash \bar{C}] .
$$

Proof. Fix $\varepsilon>0$. Let $\left\{m_{k}^{(1)}\right\} \subset S B V\left(A \backslash \bar{C} ; \mathbb{R}^{N}\right),\left\{m_{k}^{(2)}\right\} \subset S B V\left(B ; \mathbb{R}^{N}\right)$ be sequences such that

$$
\begin{aligned}
\mathcal{F}[(m, M) ; A \backslash \bar{C}]+\varepsilon \geq \lim \{ & \int_{A \backslash \bar{C}} f\left(x, m_{k}^{(1)}(x), \nabla m_{k}^{(1)}(x)\right) d x \\
& \left.+\int_{A \backslash \bar{C} \cap S\left(m_{k}^{(1)}\right)}\left|\left[m_{k}^{(1)}\right](x)\right| d \mathcal{H}^{N-1}(x)\right\},
\end{aligned}
$$

$\left|m_{k}^{(1)}(x)\right|=1$ a.e. in $A \backslash \bar{C}, m_{k}^{(1)} \rightarrow m$ in $L^{1}\left(A \backslash \bar{C} ; \mathbb{R}^{N}\right), \nabla m_{k}^{(1)} \rightarrow M$ in $L^{2}(A \backslash$ $\bar{C} ; \mathbb{R}^{N^{2}}$ ), and

$$
\begin{aligned}
\mathcal{F}[(m, M) ; B]+\varepsilon \geq \lim \{ & \int_{B} f\left(x, m_{k}^{(2)}(x), \nabla m_{k}^{(2)}(x)\right) d x \\
& \left.+\int_{B \cap S\left(m_{k}^{(2)}\right)}\left|\left[m_{k}^{(2)}\right](x)\right| d \mathcal{H}^{N-1}(x)\right\},
\end{aligned}
$$

$\left|m_{k}^{(2)}(x)\right|=1$ a.e. in $B, m_{k}^{(2)} \rightarrow m$ in $L^{1}\left(B ; \mathbb{R}^{N}\right), \nabla m_{k}^{(2)} \rightarrow M$ in $L^{2}\left(B ; \mathbb{R}^{N^{2}}\right)$.

Up to a subsequence, we can find bounded Radon measures, $\nu, \mu_{1}$ and $\mu_{2}$ such that

$$
\begin{gathered}
\left(\left|\nabla m_{k}^{(1)}\right|^{2}+\left|\nabla m_{k}^{(2)}\right|^{2}\right) \mathcal{L}^{N}\lfloor(B \backslash \bar{C}) \stackrel{*}{\rightarrow} \nu \\
\left|\left[m_{k}^{(1)}\right]\right| \mathcal{H}^{N-1}\left\lfloor\left(S\left(m_{k}^{(1)}\right) \cap(B \backslash \bar{C})\right) \stackrel{*}{\rightarrow} \mu_{1}\right. \\
\left|\left[m_{k}^{(2)}\right]\right| \mathcal{H}^{N-1}\left\lfloor\left(S\left(m_{k}^{(2)}\right) \cap(B \backslash \bar{C})\right) \rightarrow * \mu_{2}\right.
\end{gathered}
$$


Let $S_{\delta}:=\{x \in B: \operatorname{dist}(x, \partial C)<\delta\}$ and choose $\delta_{0}$ such that $\nu\left(\partial S_{\delta_{0}}\right)=$ $\mu_{1}\left(\partial S_{\delta_{0}}\right)=\mu_{2}\left(\partial S_{\delta_{0}}\right)=0$ and $\int_{\partial S_{\delta_{0}} \cap S(m)}|[m](x)| d \mathcal{H}^{N-1}=0$. Let $S:=S_{\delta_{0}}$ and set $S^{i}:=\left\{x \in B: \operatorname{dist}(x, S)<\frac{1}{i}\right\}$. We consider a family of cut-off functions $\varphi^{i} \in C^{\infty}(A ;[0,1]), \varphi^{i}(x)=1$ on $A \backslash S^{i}, \varphi^{i}=0$ in $S^{i+1},\left\|\nabla \varphi^{i}\right\|_{\infty} \leq C i^{2}$. Let

$$
m_{i, k}:=\varphi^{i} m_{k}^{(1)}+\left(1-\varphi^{i}\right) m_{k}^{(2)} .
$$

Clearly $m_{i, k} \rightarrow m$ in $L^{1}\left(A ; \mathbb{R}^{N}\right), \nabla m_{i, k} \rightarrow M$ in $L^{2}\left(A ; \mathbb{R}^{N^{2}}\right)$ as $k \rightarrow+\infty$, and now we need to modify this sequence in order to have its range on $S^{N-1}$. We will do that in two steps: first we modify the sequence $\left\{m_{i, k}\right\}$ into a new one $\left\{\bar{m}_{i, k}\right\}$ of the form

$$
\bar{m}_{i, k}(x):= \begin{cases}m_{i, k}(x) & \text { if }\left|m_{i, k}(x)\right|>\eta_{i k}, \\ m(x) & \text { otherwise }\end{cases}
$$

for suitable $\eta_{i k}$ in order to have $\left|\bar{m}_{i, k}(x)\right| \geq \eta>0$ and afterwards we project $\left\{\bar{m}_{i, k}\right\}$ onto the unit sphere by considering $\tilde{m}_{i, k}:=\Pi\left(\bar{m}_{i, k}\right)$.

We consider the Lipschitz function

$$
f(x):= \begin{cases}1 & \text { if }|x|>1 \\ |x| & \text { if } \frac{1}{2}<|x| \leq 1 \\ \frac{1}{2} & \text { if }|x| \leq \frac{1}{2}\end{cases}
$$

The composite function $w_{i, k}:=f\left(m_{i, k}\right)$ belongs to $S B V$ (see $[4,6]$ ). Set $E_{i k}^{\eta}:=$ $\left\{x \in S^{i} \backslash \overline{S^{i+1}}:\left|m_{i k}(x)\right|>\eta\right\}$. By Theorem $2.3 E_{i k}^{\eta}$ has finite perimeter for a.e. $\eta$ and all $(i, k)$ and

$$
\begin{aligned}
\int_{\frac{1}{2}}^{1} P\left(E_{i k}^{\eta} ; S^{i} \backslash \overline{S^{i+1}}\right) d \eta & =\left|D w_{i, k}\right|\left(S^{i} \backslash \overline{S^{i+1}}\right) \\
\leq & \int_{S^{i} \backslash \overline{S^{i+1}}}\left|\nabla m_{i, k}\right| d x+\left|D^{s} w_{i, k}\right|\left(S^{i} \backslash \overline{S^{i+1}}\right) \\
\leq & \int_{S^{i} \backslash \overline{S^{i+1}}}\left|\nabla m_{i, k}\right| d x \\
& \quad+\int_{\left(\overline{S^{i}} \backslash S^{i+1}\right) \cap S\left(m_{k}^{(1)}\right)}\left|\left[m_{k}^{(1)}\right](x)\right| d \mathcal{H}^{N-1} \\
& \quad+\int_{\left(\overline{S^{i}} \backslash S^{i+1}\right) \cap S\left(m_{k}^{(2)}\right)}\left|\left[m_{k}^{(2)}\right](x)\right| d \mathcal{H}^{N-1} .
\end{aligned}
$$

For every $(i, k)$ we can find numbers $\eta_{i k} \in\left(\frac{1}{2}, 1\right)$ such that $E_{i k}^{\eta_{i k}}$ has finite perimeter and

$$
P\left(E_{i k}^{\eta_{i k}} ; S^{i} \backslash \overline{S^{i+1}}\right) \leq 2 \int_{\frac{1}{2}}^{1} P\left(E_{i k}^{\eta} ; S^{i} \backslash \overline{S^{i+1}}\right) d \eta .
$$

In view of (3.7) and (3.7), it follows that

$$
\lim _{i \rightarrow+\infty} \lim _{k \rightarrow+\infty} P\left(E_{i k}^{\eta_{i k}} ; S^{i} \backslash \overline{S^{i+1}}\right)=0 .
$$


We have

$$
\begin{aligned}
& \mathcal{F}[(m, M) ; A] \\
& \leq \liminf _{i \rightarrow+\infty} \liminf _{k \rightarrow+\infty}\left\{\int_{A} f\left(x, \tilde{m}_{i, k}, \nabla \tilde{m}_{i, k}\right) d x+\int_{A \cap S\left(\tilde{m}_{i, k}\right)}\left|\left[\tilde{m}_{i, k}\right]\right| d \mathcal{H}^{N-1}\right\} \\
& \leq \lim _{k \rightarrow+\infty}\left\{\int_{A \backslash \bar{C}} f\left(x, m_{k}^{(1)}, \nabla m_{k}^{(1)}\right) d x+\int_{(A \backslash \bar{C}) \cap S\left(m_{k}^{(1)}\right)}\left|\left[m_{k}^{(1)}\right]\right| d \mathcal{H}^{N-1}\right\} \\
& +\lim _{k \rightarrow+\infty}\left\{\int_{B} f\left(x, m_{k}^{(2)}, \nabla m_{k}^{(2)}\right) d x+\int_{B \cap S\left(m_{k}^{(2)}\right)}\left|\left[m_{k}^{(2)}\right]\right| d \mathcal{H}^{N-1}\right\} \\
& +C \limsup _{i \rightarrow+\infty} \limsup _{k \rightarrow+\infty}\left\{\int_{S^{i} \backslash \overline{S^{i+1}}}\left(1+\left|\nabla \tilde{m}_{i k}\right|^{2}\right) d x\right. \\
& \left.+\int_{\left(S^{i} \backslash \overline{S^{i+1}}\right) \cap S\left(\tilde{m}_{i k}\right)}\left|\left[\tilde{m}_{i k}\right]\right| d \mathcal{H}^{N-1}\right\} \\
& \leq \mathcal{F}[(m, M) ; A \backslash \bar{C}]+\mathcal{F}[(m, M) ; B]+2 \varepsilon \\
& +C \limsup _{i \rightarrow+\infty} \limsup _{k \rightarrow+\infty}\left\{\int_{S^{i} \backslash \overline{S^{i+1}}}\left(1+\left|\nabla \bar{m}_{i k}\right|^{2}\right) d x\right. \\
& \left.+\int_{\left(S^{i} \backslash \overline{S^{i+1}}\right) \cap S\left(\bar{m}_{i k}\right)}\left|\left[\tilde{m}_{i k}\right]\right| d \mathcal{H}^{N-1}\right\} \\
& \leq \mathcal{F}[(m, M) ; A \backslash \bar{C}]+\mathcal{F}[(m, M) ; B]+2 \varepsilon \\
& +\limsup _{i \rightarrow+\infty} \limsup _{k \rightarrow+\infty}\left\{C \int_{S^{i} \backslash \overline{S^{i+1}}}\left(1+\left|\nabla m_{k}^{(1)}\right|^{2}+\left|\nabla m_{k}^{(2)}\right|^{2}\right) d x\right. \\
& +C \int_{S^{i} \backslash \overline{S^{i+1}}}\left|\nabla \varphi^{i}\right|^{2}\left|m_{k}^{(1)}-m_{k}^{(2)}\right|^{2} d x+C \int_{S^{i} \backslash \overline{S^{i+1}}}|\nabla m|^{2} d x \\
& +C \int_{\left(S^{i} \backslash \overline{S^{i+1}}\right) \cap S\left(m_{k}^{(1)}\right)}\left|\left[m_{k}^{(1)}\right]\right| d \mathcal{H}^{N-1}+C \int_{\left(S^{i} \backslash \overline{S^{i+1}}\right) \cap S\left(m_{k}^{(2)}\right)}\left|\left[m_{k}^{(2)}\right]\right| d \mathcal{H}^{N-1} \\
& \left.+C \int_{\left(S^{i} \backslash \overline{S^{i+1}}\right) \cap S(m)}|[m]| d \mathcal{H}^{N-1}+C P\left(E_{i k}^{\eta_{i k}}, A\right)\right\} .
\end{aligned}
$$

By (3.7) and (3.8) we obtain

$$
\mathcal{F}[(m, M) ; A] \leq \mathcal{F}[(m, M) ; A \backslash \bar{C}]+\mathcal{F}[(m, M) ; B]+2 \varepsilon,
$$

and since $\varepsilon$ is an arbitrary positive number, we deduce the subadditivity.

Next we prove that $\mathcal{F}[(m, M) ;$.$] is the trace on the open subsets of \Omega$ of a bounded Radon measure. The argument is exactly similar to that used in Proposition 2.22 in [8] and we include it here for the convenience of the reader. 
Proposition 3.4. There exists a bounded Radon measure $\mu$ such that

$$
\mathcal{F}[(m, M) ; A]=\mu(A)
$$

for every open set $A \subset \Omega$. Moreover $\mu<<\mathcal{L}^{N}\left\lfloor\Omega+\left|D^{s} m\right|\right.$.

Proof. We can find a sequence $\left\{m_{k}\right\}$ such that

$$
\begin{aligned}
\mathcal{F}[(m, M) ; \Omega]= & \lim _{k \rightarrow+\infty}\left\{\int_{\Omega} f\left(x, m_{k}(x), \nabla m_{k}(x)\right) d x\right. \\
& \left.+\int_{\Omega \cap S\left(m_{k}\right)}\left|\left[m_{k}\right](x)\right| d \mathcal{H}^{N-1}\right\}
\end{aligned}
$$

and

$$
f\left(x, m_{k}(x), \nabla m_{k}(x)\right) \mathcal{L}^{N}\left\lfloor\Omega+\left|\left[m_{k}\right](x)\right| \mathcal{H}^{N-1}\left\lfloor\left(\Omega \cap S\left(m_{k}\right)\right) \stackrel{*}{\rightarrow} \mu,\right.\right.
$$

where $\mu \in \mathcal{M}\left(\mathbb{R}^{N}\right)$. We note that we extended the integrands above to all $\mathbb{R}^{N}$ by zero.

Let $V \subset \subset \Omega$ be an open set, fix $\varepsilon>0$, and let $W$ be open, with $W \subset \subset V$, and $\mu(V \backslash W)<\varepsilon$. We have

$$
\begin{aligned}
\mu(V) & \leq \mu(W)+\varepsilon=\mu\left(\mathbb{R}^{N}\right)-\mu\left(\mathbb{R}^{N} \backslash W\right)+\varepsilon \\
& \leq \mathcal{F}[(m, M) ; \Omega]-\mathcal{F}[(m, M) ; \Omega \backslash \bar{W}]+\varepsilon \\
& \leq \mathcal{F}[(m, M) ; V]+\varepsilon .
\end{aligned}
$$

As $\varepsilon$ is arbitrary we conclude that $\mu(V) \leq \mathcal{F}[(m, M) ; V]$. If we only have $V$ open, $V \subset \Omega$, then consider $V^{\prime} \subset \subset V$, apply the inequality just proved to $V^{\prime}$, i.e.

$$
\mu\left(V^{\prime}\right) \leq \mathcal{F}\left[(m, M) ; V^{\prime}\right] \leq \mathcal{F}[(m, M) ; V],
$$

and then take the supremum on the left-hand-side over all such $V^{\prime}$ s.

Now we prove the reverse inequality. Given an open set $V$, there is a compact $K \subset \subset V$ such that $\left(C\left(1+|M|^{2}\right) \mathcal{L}^{N}+C|D m|\right)(V \backslash K)<\varepsilon$. Let $W$ be an open set verifying $K \subset \subset W \subset \subset V$. By (3.3) and Lemma 3.3,

$$
\mathcal{F}[(m, M) ; V] \leq \mathcal{F}[(m, M) ; W]+\mathcal{F}[(m, M) ; V \backslash K] \leq \mu(\bar{W})+\varepsilon \leq \mu(V)+\varepsilon,
$$

and letting $\varepsilon \rightarrow 0$ we get $\mathcal{F}[(m, M) ; V] \leq \mu(V)$.

Next we characterize the densities

$$
\frac{d \mathcal{F}[(m, M) ; .]}{d \mathcal{L}^{N}}, \quad \frac{d \mathcal{F}[(m, M) ; .]}{d\left(|[m]| \mathcal{H}^{N-1}\lfloor S(m))\right.}
$$

\section{Proof. STEP 1: Lower bound}

We consider a sequence $\left\{m_{k}\right\} \subset S B V\left(\Omega ; \mathbb{R}^{N}\right)$ such that $m_{k} \rightarrow m$ in $L^{1}$, $\nabla m_{k} \rightarrow M$ in $L^{2}$ and

$$
\sup _{k}\left\{\int_{\Omega} f\left(x, m_{k}(x), \nabla m_{k}(x)\right)+\int_{\Omega \cap S\left(m_{k}\right)}\left|\left[m_{k}\right](x)\right| \mathcal{H}^{N-1}(x)\right\}<+\infty .
$$


Up to the extraction of a subsequence, which we do not relabel, we assume further that

$$
\mu_{k}:=f\left(x, m_{k}(x), \nabla m_{k}(x)\right) \mathcal{L}^{N}\left\lfloor\Omega+\left|\left[m_{k}\right](x)\right| \mathcal{H}^{N-1}\left\lfloor S\left(m_{k}\right) \stackrel{*}{\rightarrow} \mu,\right.\right.
$$

where $\mu$ is a bounded Radon measure. We now decompose the measure $\mu$ relatively to $\mathcal{L}^{N}\left\lfloor\Omega\right.$ and $|[m](x)| \mathcal{H}^{N-1}\left\lfloor S(m)\right.$, denoting the respective densities by $\mu_{a}$ and $\mu_{s}$. We start by establishing a lower bound for $\mu_{a}$.

Fix $x_{0} \in \Omega$ such that

$$
\lim _{r \rightarrow 0} \frac{\mu\left(Q\left(x_{0}, r\right)\right)}{r^{N}}
$$

exists and is finite,

$$
\lim _{r \rightarrow 0} \frac{1}{r^{N+1}} \int_{Q\left(x_{0}, r\right)}\left|m(x)-m\left(x_{0}\right)-\nabla m\left(x_{0}\right)\left(x-x_{0}\right)\right| d x=0,
$$

and

$$
\lim _{r \rightarrow 0} \frac{1}{r^{N}} \int_{Q\left(x_{0}, r\right)}\left|M(x)-M\left(x_{0}\right)\right|^{2} d x=0 .
$$

The set of points that do not satisfy all these conditions has Lebesgue measure zero. have

We choose a sequence $r_{n} \rightarrow 0$ such that $\mu\left(\partial Q\left(x_{0}, r_{n}\right)\right)=0$ for all $n$, and we

$$
\begin{aligned}
\mu_{a}\left(x_{0}\right)= & \frac{d \mu}{d \mathcal{L}^{N}}\left(x_{0}\right)=\lim _{r_{n} \rightarrow 0} \frac{\mu\left(Q\left(x_{0}, r_{n}\right)\right)}{r_{n}^{N}}=\lim _{r_{n} \rightarrow 0} \frac{1}{r_{n}^{N}} \lim _{k \rightarrow+\infty} \mu_{k}\left(Q\left(x_{0}, r_{n}\right)\right) \\
= & \lim _{r_{n} \rightarrow 0} \lim _{k \rightarrow+\infty} \frac{1}{r_{n}^{N}}\left\{\int_{Q\left(x_{0}, r_{n}\right)} f\left(x, m_{k}, \nabla m_{k}\right) d x+\int_{Q\left(x_{0}, r_{n}\right) \cap S\left(m_{k}\right)}\left|\left[m_{k}\right]\right| d \mathcal{H}^{N-1}\right\} \\
= & \lim _{r_{n} \rightarrow 0} \lim _{k \rightarrow+\infty} \frac{1}{r_{n}^{N}}\left\{\int_{Q\left(x_{0}, r_{n}\right)} \bar{f}\left(x, m_{k}, \nabla m_{k}\right) d x+\int_{Q\left(x_{0}, r_{n}\right) \cap S\left(m_{k}\right)}\left|\left[m_{k}\right]\right| d \mathcal{H}^{N-1}\right\} \\
= & \lim _{r_{n} \rightarrow 0} \lim _{k \rightarrow+\infty}\left\{\int_{Q} \bar{f}\left(x_{0}+r_{n} y, m_{k}\left(x_{0}+r_{n} y\right), \nabla m_{k}\left(x_{0}+r_{n} y\right)\right) d y\right. \\
& \left.+\frac{1}{r_{n}} \int_{Q \cap \frac{S\left(m_{k}\right)-x_{0}}{r_{n}}}\left|\left[m_{k}\right]\left(x_{0}+r_{n} y\right)\right| d \mathcal{H}^{N-1}\right\}<+\infty .
\end{aligned}
$$

We introduce

$$
\omega_{k, n}(y):=\frac{m_{k}\left(x_{0}+r_{n} y\right)-m\left(x_{0}\right)}{r_{n}} .
$$

It can be easily checked that $\limsup _{n \rightarrow+\infty} \lim \sup _{k \rightarrow+\infty}\left\|\nabla \omega_{k, n}\right\|_{L^{2}} \leq C$, $\lim _{n \rightarrow+\infty} \lim _{k \rightarrow+\infty} \int_{Q}\left|\omega_{k, n}(y)-\nabla m\left(x_{0}\right) y\right| d y=0$ and $\lim _{n \rightarrow+\infty} \lim _{k \rightarrow+\infty}$ $\int_{Q}\left(\nabla \omega_{k, n}-M\left(x_{0}\right)\right) \varphi(y) d y=0$ for every $\varphi \in L^{2}$. Using a diagonalizing procedure 
and the separibility of $L^{2}$, we can find a sequence $\omega_{k} \in S B V\left(Q ; \mathbb{R}^{N}\right) \cap L^{\infty}\left(Q ; \mathbb{R}^{N}\right)$, verifying the conditions

$$
\omega_{k} \rightarrow \nabla m\left(x_{0}\right) y \text { in } L^{1}, \quad \nabla \omega_{k} \rightarrow M\left(x_{0}\right) \text { in } L^{2}
$$

and

$$
\begin{aligned}
\frac{d \mu}{d \mathcal{L}^{N}}\left(x_{0}\right) \geq & \lim _{k \rightarrow+\infty}\left\{\int_{Q} \bar{f}\left(x_{0}+r_{k} y, m\left(x_{0}\right)+r_{k} \omega_{k}(y), \nabla \omega_{k}(y)\right) d y\right. \\
& \left.+\int_{Q \cap S\left(\omega_{k}\right)}\left|\left[\omega_{k}\right](y)\right| d \mathcal{H}^{N-1}\right\} .
\end{aligned}
$$

Now we prove the existence of another sequence $\bar{\omega}_{k} \in S B V\left(Q ; \mathbb{R}^{N}\right) \cap L^{\infty}\left(Q ; \mathbb{R}^{N}\right)$, $\nabla \bar{\omega}_{k} \in L^{2}\left(Q ; \mathbb{R}^{N^{2}}\right)$, verifying the conditions

$$
\bar{\omega}_{k} \rightarrow \nabla m\left(x_{0}\right) y \text { in } L^{1}, \quad \nabla \bar{\omega}_{k} \rightarrow M\left(x_{0}\right) \text { in } L^{2}
$$

and

$$
\begin{aligned}
& \frac{d \mu}{d \mathcal{L}^{N}}\left(x_{0}\right) \geq \liminf _{k \rightarrow+\infty}\left\{\int_{Q} \bar{f}\left(x_{0}+r_{k} y, m\left(x_{0}\right)+r_{k} \omega_{k}(y), \nabla \omega_{k}(y)\right) d y\right. \\
&\left.+\int_{Q \cap S\left(\omega_{k}\right)}\left|\left[\omega_{k}\right]\right| d \mathcal{H}^{N-1}\right\} \\
& \geq \liminf _{k \rightarrow+\infty}\left\{\int_{Q} \bar{f}\left(x_{0}, m\left(x_{0}\right), \nabla \bar{\omega}_{k}(y)\right) d y+\int_{Q \cap S\left(\bar{\omega}_{k}\right)}\left|\left[\bar{\omega}_{k}\right](y)\right| d \mathcal{H}^{N-1}\right\} .
\end{aligned}
$$

To this end, we consider the family of Lipschitz continuous functions $\varphi_{i}: \mathbb{R}^{N} \rightarrow$ $\mathbb{R}^{N}$,

$$
\varphi_{i}(x):= \begin{cases}x & \text { if }|x| \leq \frac{1}{2^{i+1}} \\ -x+\frac{1}{2^{i}} \frac{x}{|x|} & \text { if } \frac{1}{2^{i+1}}<|x| \leq \frac{1}{2^{i}} \\ 0 & \text { otherwise }\end{cases}
$$

We note $\varphi_{i}$ has Lipschitz constant 1 and we define

$$
\hat{\omega}_{i, k}:=\nabla m\left(x_{0}\right) y+\varphi_{i}\left(\omega_{k}(y)-\nabla m\left(x_{0}\right) y\right) .
$$

Using the chain rule (see $[4,6]$ ) we find

$$
\nabla \hat{\omega}_{i, k}=\nabla m\left(x_{0}\right)+\nabla^{\tau} \varphi_{i}\left(\omega_{k}(y)-\nabla m\left(x_{0}\right) y\right) \cdot\left(\nabla \omega_{k}(y)-\nabla m\left(x_{0}\right)\right)
$$

and

$$
\left|D^{s} \hat{\omega}_{i, k}\right|(Q) \leq\left|D^{s} \omega_{k}\right|(Q),
$$

where $\nabla^{\tau} \varphi_{i}$ denotes $\nabla\left(\varphi_{i} \mid A(y)\right)$, with $A(y):=\tilde{\omega}_{k}(y)-\nabla m\left(x_{0}\right) y+\left\{\left(\nabla \omega_{k}(y)-\nabla m\right.\right.$ $\left.\left.\left(x_{0}\right)\right) v: v \in \mathbb{R}^{N}\right\}$ (see [6] pag. 193, Thm. 3.101). 
We have

$$
\begin{aligned}
& \int_{Q} \bar{f}\left(x_{0}+r_{k} y, m\left(x_{0}\right)+r_{k} \hat{\omega}_{i, k}(y), \nabla \hat{\omega}_{i, k}(y)\right) d y \\
& \leq \int_{Q \cap\left\{y \in Q:\left|\omega_{k}(y)-\nabla m\left(x_{0}\right) y\right| \leq \frac{1}{2^{i+1}}\right\}} \bar{f}\left(x_{0}+r_{k} y, m\left(x_{0}\right)+r_{k} \omega_{k}(y), \nabla \omega_{k}(y)\right) d y \\
& \quad+C \int_{Q \cap\left\{y \in Q: \frac{1}{2^{i+1}}<\left|\omega_{k}(y)-\nabla m\left(x_{0}\right) y\right| \leq \frac{1}{2^{i}}\right\}}\left(1+\left|\nabla \hat{\omega}_{i, k}\right|^{2}\right) d y \\
& \quad+C \int_{Q \cap\left\{y \in Q:\left|\omega_{k}(y)-\nabla m\left(x_{0}\right) y\right|>\frac{1}{2^{i}}\right\}}\left(1+\left|\nabla m\left(x_{0}\right)\right|^{2}\right) d y .
\end{aligned}
$$

We show that the last two terms tend to 0 if we choose $(i, k)$ going to infinity in a suitable way. Let $j_{k}$ be the greatest even number verifying the inequality

$$
2^{j} \leq \frac{1}{\sqrt{\left\|\omega_{k}-\nabla m\left(x_{0}\right) y\right\|_{L^{1}(Q)}}}
$$

For every $i \leq j_{k}$

$$
\begin{aligned}
\left|\left\{y \in Q:\left|\omega_{k}(y)-\nabla m\left(x_{0}\right) y\right|>\frac{1}{2^{i}}\right\}\right| & \leq 2^{i} \int_{Q}\left|\omega_{k}-\nabla m\left(x_{0}\right) y\right| d y \\
& \leq \sqrt{\left\|\omega_{k}-\nabla m\left(x_{0}\right) y\right\|_{L^{1}(Q)}} \rightarrow 0
\end{aligned}
$$

as $k \rightarrow+\infty$. From the bound

$$
\begin{aligned}
& \sum_{i=\frac{j_{k}}{2}}^{j_{k}} \int_{Q \cap\left\{y \in Q: \frac{1}{2^{i+1}} \leq\left|\omega_{k}(y)-\nabla m\left(x_{0}\right) y\right|<\frac{1}{2^{i}}\right\}} C\left(1+\left|\nabla \hat{\omega}_{i, k}\right|^{2}\right) d y \\
& \quad \leq C \int_{Q}\left(1+\left|\nabla \omega_{k}(y)-\nabla m\left(x_{0}\right)\right|^{2}\right) d y \leq C,
\end{aligned}
$$

we get the existence of an index $i_{k} \in\left[\frac{j_{k}}{2}, j_{k}\right]$ such that

$$
\int_{Q \cap\left\{y \in Q: \frac{1}{2^{i} k^{+1}} \leq\left|\omega_{k}(y)-\nabla m\left(x_{0}\right) y\right|<\frac{1}{2^{i} k}\right\}} C\left(1+\left|\nabla \hat{\omega}_{i_{k}, k}\right|^{2}\right) d y \leq \frac{2 C}{j_{k}+2} .
$$

We define $\bar{\omega}_{k}:=\hat{\omega}_{i_{k}, k}$, and it is easy to check that

$$
\bar{\omega}_{k} \rightarrow \nabla m\left(x_{0}\right) y \text { in } L^{\infty}, \quad \nabla \bar{\omega}_{k} \rightarrow M\left(x_{0}\right) \text { in } L^{2},
$$

and, by (3.11) and (3.12),

$$
\begin{aligned}
& \liminf _{k \rightarrow+\infty} \int_{Q} \bar{f}\left(x_{0}+r_{k} y, m\left(x_{0}\right)+r_{k} \bar{\omega}_{k}(y), \nabla \bar{\omega}_{k}(y)\right) d y \\
& \quad \leq \liminf _{k \rightarrow+\infty} \int_{Q} \bar{f}\left(x_{0}+r_{k} y, m\left(x_{0}\right)+r_{k} \omega_{k}(y), \nabla \omega_{k}(y)\right) d y
\end{aligned}
$$


Now using hypothese $(H 2),(H 3)$, and by (3.7), we get the inequality $(3.7)$, which, in turn, yields

$$
\mu_{a}\left(x_{0}\right) \geq H\left(x_{0}, m\left(x_{0}\right), \nabla m\left(x_{0}\right), M\left(x_{0}\right)\right) \text { for } \mathcal{L}^{N} \text { a.e. } x \text { in } \Omega .
$$

Next we obtain a lower bound for the density $\mu_{s}$. Fix $x_{0} \in S(m)$ such that

$$
\lim _{r \rightarrow 0} \frac{\mu\left(Q\left(x_{0}, r\right)\right)}{\int_{Q\left(x_{0}, r\right) \cap S(m)}|[m](x)| d \mathcal{H}^{N-1}}
$$

exists and is finite. The complementar to this set of points in $S(m)$ has zero $|[m]| \mathcal{H}^{N-1}\lfloor S(m)$-measure. We have

$$
\begin{aligned}
\mu_{s}\left(x_{0}\right) & =\frac{d \mu}{d\left(|[m](x)| \mathcal{H}^{N-1}\lfloor S(m))\right.}=\lim _{r_{n} \rightarrow 0} \frac{\mu\left(Q\left(x_{0}, r_{n}\right)\right)}{\int_{Q\left(x_{0}, r_{n}\right) \cap S(m)}|[m]| d \mathcal{H}^{N-1}} \\
& =\lim _{r_{n} \rightarrow 0} \lim _{k \rightarrow+\infty} \frac{\mu_{k}\left(Q\left(x_{0}, r_{n}\right)\right)}{\int_{Q\left(x_{0}, r_{n}\right) \cap S(m)}|[m]| d \mathcal{H}^{N-1}} \\
& =\lim _{r_{n} \rightarrow 0} \lim _{k \rightarrow+\infty}\left\{\frac{\int_{Q\left(x_{0}, r_{n}\right)} f\left(x, m_{k}, \nabla m_{k}\right) d x}{\int_{Q\left(x_{0}, r_{n}\right) \cap S(m)}|[m]| d \mathcal{H}^{N-1}}+\frac{\int_{Q\left(x_{0}, r_{n}\right) \cap S\left(m_{k}\right)}\left|\left[m_{k}\right]\right| d \mathcal{H}^{N-1}}{\int_{Q\left(x_{0}, r_{n}\right) \cap S(m)}|[m]| d \mathcal{H}^{N-1}}\right\} \\
& \geq \liminf _{r_{n} \rightarrow 0} \liminf _{k \rightarrow+\infty} \frac{\int_{Q\left(x_{0}, r_{n}\right) \cap S\left(m_{k}\right)}\left|\left[m_{k}\right]\right| d \mathcal{H}^{N-1}}{\int_{Q\left(x_{0}, r_{n}\right) \cap S(m)}|[m]| d \mathcal{H}^{N-1}} \\
& \geq \liminf _{r_{n} \rightarrow 0} \frac{\int_{Q\left(x_{0}, r_{n}\right)}|\nabla m-M| d x+\int_{Q\left(x_{0}, r_{n}\right) \cap S(m)}|[m](x)| d \mathcal{H}^{N-1}}{\int_{Q\left(x_{0}, r_{n}\right) \cap S(m)}|[m]| d \mathcal{H}^{N-1}} \geq 1,
\end{aligned}
$$

where we have used the fact that $\left[m_{k}\right] \mathcal{H}^{N-1}\left\lfloor S\left(m_{k}\right) \stackrel{*}{\rightarrow}(\nabla m-M) \mathcal{L}^{N}\left\lfloor\Omega+[m] \mathcal{H}^{N-1}\right.\right.$ $\left\lfloor S\left(m_{k}\right)\right.$ and the lower semicontinuity of the total variation with respect to weak ${ }^{*}$ convergence. This together with (3.13), entails

$$
\begin{aligned}
& \liminf _{k \rightarrow+\infty} \int_{\Omega} f\left(x, m_{k}(x), \nabla m_{k}(x)\right) d x+\int_{\Omega \cap S\left(m_{k}\right)}\left|\left[m_{k}\right](x)\right| \mathcal{H}^{N-1} \\
& \geq \mu(\Omega) \geq \int_{\Omega} H(x, m(x), \nabla m(x), M(x)) d x+\int_{\Omega \cap S(m)}|[m](x)| d \mathcal{H}^{N-1}
\end{aligned}
$$

\section{STEP 2: Upper bound}

Fix $(m, M)$. As the function $(x, v) \rightarrow f(x, m(x), v)$ is Carathéodory, using Scorza-Dragoni Theorem, we can find sets $K_{j}$ such that $\left|\Omega \backslash K_{j}\right|<\frac{1}{j}$ and $(x, v) \rightarrow$ $f(x, m(x), v)$ is continuous on $K_{j} \times \mathbb{R}^{N^{2}}$. We denote by $K_{j}^{*}$ the set of Lebesgue points of $\chi_{K_{j}}$ and we define $\omega:=\cup\left(K_{j} \cap K_{j}^{*}\right)$. Fix $x_{0} \in \omega$ a Lebesgue point for $m, \nabla m$ and $M$.

Let $a:=m\left(x_{0}\right), A:=\nabla m\left(x_{0}\right), B:=M\left(x_{0}\right)$. Given $\delta>0$ we can find $u \in S B V\left(Q ; \mathbb{R}^{N}\right) \cap L^{\infty}, \nabla u \in L^{2},\left.u\right|_{\partial Q}=A x, \int_{Q} \nabla u(x) d x=B$, such that

$$
\int_{Q} f\left(x_{0}, a, \nabla u(x)\right) d x+\int_{Q \cap S(u)}|[u](x)| \mathcal{H}^{N-1} \leq H\left(x_{0}, a, A, B\right)+\delta .
$$


We write

$$
u(x)=A x+\Phi(x),
$$

where $\left.\Phi\right|_{\partial Q}=0, \int_{Q} \nabla \Phi(x) d x=B-A$ and $\Phi$ is extended to all $\mathbb{R}^{N}$ by periodicity. We define

$$
m_{\varepsilon, n}:=\Pi\left(m(x)+\frac{\varepsilon}{n} \Phi_{\varepsilon, n}(x)+h_{\varepsilon}-h_{\varepsilon, n}\right), \quad x \in Q\left(x_{0}, \varepsilon\right)
$$

where $\Phi_{\varepsilon, n}(x):=\Phi\left(\frac{n\left(x-x_{0}\right)}{\varepsilon}\right), h_{\varepsilon} \in S B V\left(Q\left(x_{0}, \varepsilon\right) ; \mathbb{R}^{N}\right)$ is such that

$$
\nabla h_{\epsilon}=M(x)-\nabla m(x)+\nabla m\left(x_{0}\right)-M\left(x_{0}\right),
$$

(see Theorem 2.11)

$$
\left|D h_{\varepsilon}\right|\left(Q\left(x_{0}, \varepsilon\right)\right) \leq C \int_{Q\left(x_{0}, \varepsilon\right)}\left|M(x)-M\left(x_{0}\right)-\nabla m(x)+\nabla m\left(x_{0}\right)\right| d x,
$$

and $h_{\varepsilon, n} \in S B V\left(Q\left(x_{0}, \varepsilon\right)\right)$ are such that

$$
\nabla h_{\varepsilon, n}=0, \quad h_{\varepsilon, n}-h_{\varepsilon} \rightarrow 0 \text { in } L^{\infty}, \quad\left|D h_{\varepsilon, n}\right|\left(Q\left(x_{0}, \varepsilon\right)\right) \rightarrow\left|D h_{\varepsilon}\right|\left(Q\left(x_{0}, \varepsilon\right)\right)
$$

(see Theorem 2.12).

We note that for fixed $\varepsilon$ and $n$ large enough $m_{\varepsilon, n}$ is well defined, $m_{\varepsilon, n} \in$ $S B V\left(Q\left(x_{0}, \varepsilon\right) ; \mathbb{R}^{N}\right),\left|m_{\varepsilon, n}(x)\right|=1$ a.e., $m_{\varepsilon, n} \rightarrow m$ in $L^{\infty}, \nabla m_{\varepsilon, n} \rightarrow M$ in $L^{2}$ and $\left\{\nabla m_{\varepsilon, n}\right\}$ is 2-equi-integrable. We then have

$$
\begin{aligned}
\mathcal{F}\left[(m, M) ; Q\left(x_{0}, \varepsilon\right)\right] \leq & \liminf _{n \rightarrow+\infty}\left\{\int_{Q\left(x_{0}, \varepsilon\right)} f\left(x, m_{\varepsilon, n}(x), \nabla m_{\varepsilon, n}(x)\right) d x\right. \\
& \left.+\int_{Q\left(x_{0}, \varepsilon\right) \cap S\left(m_{\varepsilon, n}\right)}\left|\left[m_{\varepsilon, n}\right](x)\right| \mathcal{H}^{N-1}(x)\right\} .
\end{aligned}
$$

We start by treating the volume part, and we prove that

$$
\begin{aligned}
& \limsup _{\varepsilon \rightarrow 0} \limsup _{n \rightarrow+\infty} \frac{1}{\varepsilon^{N}} \int_{Q\left(x_{0}, \varepsilon\right)} f\left(x, m_{\varepsilon, n}(x), \nabla m_{\varepsilon, n}(x)\right) d x \\
& \quad \leq \limsup \limsup _{\epsilon \rightarrow 0} \frac{1}{\epsilon^{N}} \int_{Q\left(x_{0}, \epsilon\right)} f\left(x, m(x), \nabla m_{\epsilon, n}(x)\right) d x .
\end{aligned}
$$

For fixed $\varepsilon$ and using the 2-equi-integrability of $\left\{\nabla m_{\varepsilon, n}\right\}$, for each $\delta^{\prime}>0$ we can find $\gamma>0$ such that

$$
\int_{Q\left(x_{0}, \varepsilon\right) \cap A} C\left(1+\left|\nabla m_{\varepsilon, n}(x)\right|^{2}\right) d x<\delta^{\prime} \varepsilon^{N}
$$

whenever $|A|<\gamma$, where $C$ comes from (H1). Since $f$ is Carathéodory, find a compact set $K_{\epsilon}$, with $\left|Q\left(x_{0}, \varepsilon\right) \backslash K_{\varepsilon}\right|<\gamma$, such that $f \mid K_{\varepsilon} \times \mathbb{R}^{N} \times \mathbb{R}^{N^{2}}$ is continuous, and define

$$
E_{\epsilon, n}:=\left\{x \in Q\left(x_{0}, \epsilon\right):\left|\nabla m_{\epsilon, n}(x)\right|<L\right\},
$$


where $L$ is large enough to guarantee that $\left|Q\left(x_{0}, \varepsilon\right) \backslash E_{\varepsilon, n}^{c}\right|<\gamma$. For $x \in Q\left(x_{0}, \varepsilon\right) \cap$ $K_{\varepsilon} \cap E_{\varepsilon, n}$ we have

$$
\left|f\left(x, m_{\varepsilon, n}(x), \nabla m_{\varepsilon, n}(x)\right)-f\left(x, m(x), \nabla m_{\varepsilon, n}(x)\right)\right|<\delta^{\prime},
$$

for $n$ large enough because of the uniform continuity of $f$ on compact subsets of $K_{\varepsilon} \times \mathbb{R}^{N} \times \mathbb{R}^{N^{2}}$ and the $L^{\infty}$ convergence of $m_{\varepsilon, n}$ to $m$.

We then have

$$
\begin{aligned}
\int_{Q\left(x_{0}, \varepsilon\right)} f( & \left.x, m_{\varepsilon, n}(x), \nabla m_{\varepsilon, n}(x)\right) d x \\
= & \int_{Q\left(x_{0}, \varepsilon\right) \cap K_{\varepsilon}} f\left(x, m_{\varepsilon, n}(x), \nabla m_{\varepsilon, n}(x)\right) d x \\
& +\int_{Q\left(x_{0}, \varepsilon\right) \backslash K_{\varepsilon}} f\left(x, m_{\varepsilon, n}(x), \nabla m_{\varepsilon, n}(x)\right) d x \\
\leq & \int_{Q\left(x_{0}, \varepsilon\right) \cap K_{\varepsilon}} f\left(x, m_{\varepsilon, n}(x), \nabla m_{\varepsilon, n}(x)\right) d x+\delta^{\prime} \varepsilon^{N} \\
= & \int_{Q\left(x_{0}, \varepsilon\right) \cap K_{\varepsilon} \cap E_{\varepsilon, n}} f\left(x, m_{\varepsilon, n}(x), \nabla m_{\varepsilon, n}(x)\right) d x \\
& +\int_{Q\left(x_{0}, \varepsilon\right) \cap K_{\varepsilon} \cap E_{\varepsilon, n}^{c}} f\left(x, m_{\varepsilon, n}(x), \nabla m_{\varepsilon, n}(x)\right) d x+\delta^{\prime} \varepsilon^{N} \\
\leq & \int_{Q\left(x_{0}, \varepsilon\right) \cap K_{\varepsilon} \cap E_{\varepsilon, n}} f\left(x, m^{N}(x), \nabla m_{\varepsilon, n}(x)\right) d x+3 \delta^{\prime} \varepsilon^{N} \\
\leq & \int_{Q\left(x_{0}, \varepsilon\right)} f\left(x, m(x), \nabla m_{\varepsilon, n}(x)\right) d x+3 \delta^{\prime} \varepsilon^{N} .
\end{aligned}
$$

Dividing through by $\varepsilon^{N}$, we have

$$
\begin{aligned}
& \limsup _{\varepsilon \rightarrow 0} \limsup _{n \rightarrow+\infty} \frac{1}{\varepsilon^{N}} \int_{Q\left(x_{0}, \varepsilon\right)} f\left(x, m_{\varepsilon, n}(x), \nabla m_{\varepsilon, n}(x)\right) d x \\
& \quad \leq \limsup _{\varepsilon \rightarrow 0} \limsup _{n \rightarrow+\infty} \frac{1}{\varepsilon^{N}} \int_{Q\left(x_{0}, \varepsilon\right)} f\left(x, m(x), \nabla m_{\varepsilon, n}(x)\right) d x+3 \delta^{\prime},
\end{aligned}
$$

and letting $\delta^{\prime} \rightarrow 0$, we deduce (3.17). Now we prove that

$$
\begin{aligned}
\limsup _{\varepsilon \rightarrow 0} & \limsup _{n \rightarrow+\infty} \frac{1}{\varepsilon^{N}} \int_{Q\left(x_{0}, \epsilon\right)} f\left(x, m(x), \nabla m_{\epsilon, n}(x)\right) d x \\
\leq & \limsup _{\varepsilon \rightarrow 0} \limsup _{n \rightarrow+\infty} \frac{1}{\varepsilon^{N}} \int_{Q\left(x_{0}, \varepsilon\right)} f\left(x, m(x), \nabla \Pi\left(m(x)+\frac{\epsilon}{n} \Phi_{\epsilon, n}(x)+h_{\epsilon}(x)\right.\right. \\
& \left.-h_{\epsilon, n}(x)\right)\left(\nabla \Phi\left(\frac{n\left(x-x_{0}\right)}{\epsilon}\right)+\nabla m\left(x_{0}\right)\right) d x .
\end{aligned}
$$


This follows from (H3), which allow us to assert that the error in passing from the left integral to the right integral, for $\varepsilon$ fixed and $n$ large enough, is given by

$$
\begin{aligned}
& \frac{C}{\epsilon^{N}} \int_{Q\left(x_{0}, \epsilon\right)}\left(1+\left|M(x)+\nabla \Phi\left(\frac{n\left(x-x_{0}\right)}{\epsilon}\right)+\nabla m\left(x_{0}\right)-M\left(x_{0}\right)\right|\right. \\
& \left.\quad+\left|\nabla \Phi\left(\frac{n\left(x-x_{0}\right)}{\epsilon}\right)+\nabla m\left(x_{0}\right)\right|\right)\left|M(x)-M\left(x_{0}\right)\right| d x .
\end{aligned}
$$

Using the fact that $x_{0}$ is a 2-Lebesgue point for $M$, Holder's inequality and Riemman Lebesgue lemma, we get that $\lim \sup _{\varepsilon \rightarrow 0} \lim \sup _{n \rightarrow+\infty}(3.18)=0$.

Using again (H3) and the $L^{\infty}$ convergence of $m(x)+\frac{\varepsilon}{n} \Phi_{\varepsilon, n}(x)+h_{\varepsilon}-h_{\varepsilon, n}$ to $m(x)$, we deduce that

$$
\begin{aligned}
& \limsup _{\varepsilon \rightarrow 0} \limsup _{n \rightarrow+\infty} \frac{1}{\varepsilon^{N}} \int_{Q\left(x_{0}, \varepsilon\right)} f\left(x, m(x), \nabla \Pi\left(m(x)+\frac{\varepsilon}{n} \Phi_{\varepsilon, n}(x)+h_{\varepsilon}(x)-h_{\varepsilon, n}(x)\right)\right. \\
& \left.\quad \times\left(\nabla \Phi\left(\frac{n\left(x-x_{0}\right)}{\varepsilon}\right)+\nabla m\left(x_{0}\right)\right)\right) d x \\
& \leq \limsup _{\varepsilon \rightarrow 0} \limsup _{n \rightarrow+\infty} \frac{1}{\varepsilon^{N}} \int_{Q\left(x_{0}, \varepsilon\right)} f(x, m(x), \nabla \Pi(m(x)) \\
& \left.\quad \times\left(\nabla \Phi\left(\frac{n\left(x-x_{0}\right)}{\varepsilon}\right)+\nabla m\left(x_{0}\right)\right)\right) d x .
\end{aligned}
$$

Now using (H3) and the fact that $x_{0}$ is a Lebesgue point for $\nabla m$, Holder's inequality and Riemman Lebesgue lemma, we get

$$
\begin{aligned}
& \limsup _{\varepsilon \rightarrow 0} \limsup _{n \rightarrow+\infty} \frac{1}{\varepsilon^{N}} \int_{Q\left(x_{0}, \varepsilon\right)} f(x, m(x), \nabla \Pi(m(x)) \\
& \left.\quad \times\left(\nabla \Phi\left(\frac{n\left(x-x_{0}\right)}{\varepsilon}\right)+\nabla m\left(x_{0}\right)\right)\right) d x \\
& \leq \limsup _{\varepsilon \rightarrow 0} \limsup _{n \rightarrow+\infty} \frac{1}{\varepsilon^{N}} \int_{Q\left(x_{0}, \varepsilon\right)} f\left(x, m(x), \nabla \Pi\left(m\left(x_{0}\right)\right)\right. \\
& \left.\quad \times\left(\nabla \Phi\left(\frac{n\left(x-x_{0}\right)}{\varepsilon}\right)+\nabla m\left(x_{0}\right)\right)\right) d x
\end{aligned}
$$


We next prove that

$$
\begin{aligned}
& \limsup _{\varepsilon \rightarrow 0} \limsup _{n \rightarrow+\infty} \frac{1}{\varepsilon^{N}} \int_{Q\left(x_{0}, \varepsilon\right)} f\left(x, m(x), \nabla \Pi\left(m\left(x_{0}\right)\right)\right. \\
& \left.\quad \times\left(\nabla \Phi\left(\frac{n\left(x-x_{0}\right)}{\varepsilon}\right)+\nabla m\left(x_{0}\right)\right)\right) d x \\
& \leq \limsup _{\epsilon \rightarrow 0} \limsup _{n \rightarrow+\infty} \frac{1}{\epsilon^{N}} \int_{Q\left(x_{0}, \epsilon\right)} f\left(x_{0}, m\left(x_{0}\right), \nabla \Pi\left(m\left(x_{0}\right)\right)\right. \\
& \left.\quad \times\left(\nabla \Phi\left(\frac{n\left(x-x_{0}\right)}{\varepsilon}\right)+\nabla m\left(x_{0}\right)\right)\right) d x
\end{aligned}
$$

Let $j_{0}$ be such that $x_{0} \in K_{j_{0}} \cap K_{j_{0}}^{*}$. We define the set

$$
E_{\varepsilon, n}:=\left\{x \in Q\left(x_{0}, \varepsilon\right):\left|\nabla \Pi\left(m\left(x_{0}\right)\right)\left(\nabla \Phi\left(\frac{n\left(x-x_{0}\right)}{\varepsilon}\right)+\nabla m\left(x_{0}\right)\right)\right|<L\right\},
$$

where $L>0$ will be defined latter. We have

$$
\begin{aligned}
\left|Q\left(x_{0}, \varepsilon\right) \backslash E_{\varepsilon, n}\right| & \leq \int_{Q\left(x_{0}, \varepsilon\right)} \frac{\left|\nabla \Pi\left(m\left(x_{0}\right)\right)\left(\nabla \Phi\left(\frac{n\left(x-x_{0}\right)}{\varepsilon}\right)+\nabla m\left(x_{0}\right)\right)\right|}{L} d x \\
& \leq \frac{C^{\prime}}{L} \varepsilon^{N} \int_{Q}\left|\nabla \Phi(n y)+\nabla m\left(x_{0}\right)\right| d y \leq \frac{C^{\prime} \varepsilon^{N}}{L} .
\end{aligned}
$$

As

$$
\begin{aligned}
& \frac{1}{\epsilon^{N}} \int_{Q\left(x_{0}, \varepsilon\right) \backslash E_{\varepsilon, n}} C\left(1+\left|\nabla \Pi\left(m\left(x_{0}\right)\right)\left(\nabla \Phi\left(\frac{n\left(x-x_{0}\right)}{\varepsilon}\right)+\nabla m\left(x_{0}\right)\right)\right|^{2}\right) d x \\
& \quad=\int_{Q \backslash \frac{E_{\varepsilon, n}-x_{0}}{\varepsilon}} C\left(1+\mid \nabla \Pi\left(\left.m\left(x_{0}\right)\left(\nabla \Phi(n y)+\nabla m\left(x_{0}\right)\right)\right|^{2}\right) d y,\right.
\end{aligned}
$$

and because of $\left|Q \backslash \frac{E_{\varepsilon, n}-x_{0}}{\varepsilon}\right|<\frac{C^{\prime}}{L}$, we can take $L$ large enough and use the 2-equi-integrability to get the estimate (independent of $\varepsilon$ )

$$
\frac{1}{\varepsilon^{N}} \int_{Q\left(x_{0}, \varepsilon\right) \backslash E_{\varepsilon, n}} C\left(1+\left|\nabla \Pi\left(m\left(x_{0}\right)\right)\left(\nabla \Phi\left(\frac{n\left(x-x_{0}\right)}{\varepsilon}\right)+\nabla m\left(x_{0}\right)\right)\right|^{2}\right) d x<\delta^{\prime} .
$$

Also, using the fact that $x_{0}$ is a Lebesgue point for $\chi_{K_{j_{0}}}$ we have $\left|\frac{Q\left(x_{0}, \varepsilon\right) \backslash K_{j_{0}}}{\varepsilon^{N}}\right| \rightarrow 0$, and an argument similar to the one above yields

$$
\frac{1}{\varepsilon^{N}} \int_{Q\left(x_{0}, \varepsilon\right) \backslash K_{j_{0}}} C\left(1+\left|\nabla \Pi\left(m\left(x_{0}\right)\right)\left(\nabla \Phi\left(\frac{n\left(x-x_{0}\right)}{\varepsilon}\right)+\nabla m\left(x_{0}\right)\right)\right|^{2}\right) d x<\delta^{\prime} .
$$

We can use the uniform continuity of $f \mid K_{j_{0}} \times \overline{B(0, L)}$ to ensure that for $\varepsilon$ small enough we have

$$
\left|f(x, m(x), v)-f\left(x_{0}, m\left(x_{0}\right), v\right)\right|<\delta^{\prime} \quad \text { for }|v| \leq L .
$$


We deduce that

$$
\begin{aligned}
\frac{1}{\varepsilon^{N}} & \int_{Q\left(x_{0}, \varepsilon\right)} f\left(x, m(x), \nabla \Pi\left(m\left(x_{0}\right)\right)\left(\nabla \Phi\left(\frac{n\left(x-x_{0}\right)}{\varepsilon}\right)+\nabla m\left(x_{0}\right)\right)\right) \\
\leq & \frac{1}{\varepsilon^{N}} \int_{Q\left(x_{0}, \varepsilon\right) \cap K_{j_{0}}} f\left(x, m(x), \nabla \Pi\left(m\left(x_{0}\right)\right)\left(\nabla \Phi\left(\frac{n\left(x-x_{0}\right)}{\varepsilon}\right)+\nabla m\left(x_{0}\right)\right)\right) \\
& +\frac{1}{\varepsilon^{N}} \int_{Q\left(x_{0}, \varepsilon\right) \backslash K_{j_{0}}} f\left(x, m(x), \nabla \Pi\left(m\left(x_{0}\right)\right)\left(\nabla \Phi\left(\frac{n\left(x-x_{0}\right)}{\varepsilon}\right)+\nabla m\left(x_{0}\right)\right)\right) \\
\leq & \frac{1}{\varepsilon^{N}} \int_{Q\left(x_{0}, \varepsilon\right) \cap K_{j_{0}} \cap E_{\varepsilon, n}} f\left(x, m(x), \nabla \Pi\left(m\left(x_{0}\right)\right)\left(\nabla \Phi\left(\frac{n\left(x-x_{0}\right)}{\varepsilon}\right)+\nabla m\left(x_{0}\right)\right)\right) \\
& +2 \delta^{\prime} \\
\leq & \frac{1}{\varepsilon^{N}} \int_{Q\left(x_{0}, \varepsilon\right) \cap K_{j_{0}} \cap E_{\varepsilon, n}} f\left(x_{0}, m\left(x_{0}\right), \nabla \Pi\left(m\left(x_{0}\right)\right)\right. \\
& \left.\times\left(\nabla \Phi\left(\frac{n\left(x-x_{0}\right)}{\varepsilon}\right)+\nabla m\left(x_{0}\right)\right)\right)+3 \delta^{\prime} \\
\leq & \frac{1}{\varepsilon^{N}} \int_{Q\left(x_{0}, \varepsilon\right)} f\left(x_{0}, m\left(x_{0}\right), \nabla\left(m\left(x_{0}\right)\right)\left(\nabla \Phi\left(\frac{n\left(x-x_{0}\right)}{\varepsilon}\right)+\nabla m\left(x_{0}\right)\right)\right)+3 \delta^{\prime},
\end{aligned}
$$

Taking limits in both sides, and in view of the fact that $\delta^{\prime}$ is arbitrary positive number, we reach the inequality (3.19). Changing variables and using RiemannLebesgue lemma

$$
\begin{aligned}
& \underset{\varepsilon \rightarrow 0}{\limsup } \limsup _{n \rightarrow+\infty} \frac{1}{\epsilon^{N}} \int_{Q\left(x_{0}, \epsilon\right)} f\left(x_{0}, m\left(x_{0}\right), \nabla \Pi\left(m\left(x_{0}\right)\right)\left(\nabla \Phi\left(\frac{n\left(x-x_{0}\right)}{\epsilon}\right)+\nabla m\left(x_{0}\right)\right)\right) \\
& =\limsup _{n \rightarrow+\infty} \int_{Q} f\left(x_{0}, m\left(x_{0}\right), \nabla \Pi\left(m\left(x_{0}\right)\right)\left(\nabla \Phi(n y)+\nabla m\left(x_{0}\right)\right) d y\right. \\
& =\int_{Q} f\left(x_{0}, m\left(x_{0}\right), \nabla \Pi\left(m\left(x_{0}\right)\right)\left(\nabla \Phi(y)+\nabla m\left(x_{0}\right)\right)\right) d y \\
& =\int_{Q} f\left(x_{0}, m\left(x_{0}\right), \nabla \Pi\left(m\left(x_{0}\right)\right) \nabla u(y)\right) d y=\int_{Q} \bar{f}\left(x_{0}, m\left(x_{0}\right), \nabla u(y)\right) d y .
\end{aligned}
$$

We now estimate the surface integral. Given $\delta^{\prime}>0$, choose $\eta>0$ small enough so that the Lipschitz constant of $\Pi \mid N_{\eta}$, where $N_{\eta}:=\left\{x \in \mathbb{R}^{N}: 1-\eta<|x|<1+\eta\right\}$, is smaller than $1+\delta^{\prime}$, we then have 


$$
\begin{aligned}
\limsup _{\varepsilon \rightarrow 0} \limsup _{n \rightarrow+\infty} \frac{1}{\varepsilon^{N}} \int_{Q\left(x_{0}, \varepsilon\right) \cap S\left(m_{\varepsilon, n}\right)}\left|\left[m_{\varepsilon, n}\right](x)\right| d \mathcal{H}^{N-1} \\
\leq \limsup _{\varepsilon \rightarrow 0} \limsup _{n \rightarrow+\infty} \frac{1+\delta^{\prime}}{\varepsilon^{N}}\left[\int_{Q\left(x_{0}, \varepsilon\right) \cap S(m)}|[m](x)| d \mathcal{H}^{N-1}\right. \\
\quad+\frac{\varepsilon}{n} \int_{Q\left(x_{0}, \varepsilon\right) \cap S\left(\Phi\left(\frac{n\left(-x_{0}\right)}{\varepsilon}\right)\right)}\left|[\Phi]\left(\frac{n\left(x-x_{0}\right)}{\varepsilon}\right)\right| d \mathcal{H}^{N-1} \\
\left.+\left|D h_{\varepsilon}\right|\left(Q\left(x_{0}, \varepsilon\right)\right)+\left|D h_{\varepsilon, n}\right|\left(Q\left(x_{0}, \varepsilon\right)\right)\right] .
\end{aligned}
$$

Using the fact that $\lim _{\varepsilon \rightarrow 0} \frac{1}{\varepsilon^{N}} \int_{Q\left(x_{0}, \varepsilon\right) \cap S(m)}|[m](x)| d \mathcal{H}^{N-1}=0$ a.e., a change of variables and the periodicity of $\Phi$ for the second term, (3.15), (3.16), and the arbitrariness of $\delta^{\prime}$, yield the estimate

$$
\limsup _{\varepsilon \rightarrow 0} \limsup _{n \rightarrow+\infty} \frac{1}{\varepsilon^{N}} \int_{Q\left(x_{0}, \varepsilon\right) \cap S\left(m_{\varepsilon, n}\right)}\left|\left[m_{\varepsilon, n}\right](x)\right| \mathcal{H}^{N-1} \leq \int_{Q \cap S(u)}|[u](x)| \mathcal{H}^{N-1} .
$$

Putting together the estimates for the volume and the surface integral, we conclude that

$$
\begin{aligned}
& \lim _{\varepsilon \rightarrow 0} \frac{\mathcal{F}\left[(m, M) ; Q\left(x_{0}, \varepsilon\right)\right] \leq}{\varepsilon^{N}} \liminf _{\varepsilon \rightarrow 0} \frac{1}{\varepsilon^{N}} \liminf _{n \rightarrow+\infty}\left\{\int_{Q\left(x_{0}, \varepsilon\right)} f\left(x, m_{\varepsilon, n}(x), \nabla m_{\varepsilon, n}\right) d x\right. \\
&\left.+\int_{Q\left(x_{0}, \varepsilon\right) \cap S\left(m_{\varepsilon, n}\right)}\left|\left[m_{\varepsilon, n}\right](x)\right| d \mathcal{H}^{N-1}\right\} \\
& \leq \limsup _{\varepsilon \rightarrow 0} \limsup _{n \rightarrow+\infty} \frac{1}{\varepsilon^{N}} \int_{Q\left(x_{0}, \varepsilon\right)} f\left(x, m_{\varepsilon, n}(x), \nabla m_{\varepsilon, n}\right) d x \\
&+\limsup _{\varepsilon^{N}} \limsup _{n \rightarrow+\infty} \frac{1}{\varepsilon^{N}} \int_{Q\left(x_{0}, \varepsilon\right) \cap S\left(m_{\varepsilon, n}\right)}\left|\left[m_{\varepsilon, n}\right](x)\right| d \mathcal{H}^{N-1} \\
& \leq \int_{Q} \bar{f}\left(x_{0}, m\left(x_{0}\right), \nabla u(y)\right) d y+\int_{Q \cap S(u)}|[u](y)| d \mathcal{H}^{N-1} \\
& \leq H\left(x_{0}, m\left(x_{0}\right), \nabla m\left(x_{0}\right), M\left(x_{0}\right)\right)+\delta,
\end{aligned}
$$

letting $\delta \rightarrow 0$, we obtain the upper bound for $\frac{d \mathcal{F}[(m, M) ; .]}{d \mathcal{L}^{N}}$.

The upper bound for the singular part of the measure $\mathcal{F}[(m, M) ;$.$] follows$ immediately from the bound (3.3)

\section{Acknowledgements}

I am very grateful to Professor Irene Fonseca for proposing this problem, for her constant support and for some helpful conversations. My research was partially funded by Fundação para a Ciência e para a Tecnologia (Grant PRAXIS XXI BD/15642/98). I also would like to thank the Center for Nonlinear Analysis (NSF Grant No. DMS-9803791), Carnegie Mellon University, Pittsburgh 
and Departamento de Matemática, Instituto Superior Técnico, Lisbon, for their support during the preparation of this paper.

\section{References}

[1] Acerbi, E., Fonseca, I., Mingione, G.: Existence and regularity for mixtures of micromagnetic materials. Proc. R. Soc. London A, 2072, 2225-2243 (2006)

[2] Alberti, G.: A Lusin type theorem for gradients. J. Funct. Anal. 100, 110-118 (1991)

[3] Ambrosio, L.: Compactness for a special case of functions of bounded variation. Boll. Un. Mat. Ital. 3B 7, 857-881 (1989)

[4] Ambrosio, L., Dal Maso, G.: A general chain-rule for distributional derivatives. Proc. Am. Math. Soc. 108, 691-702 (1990)

[5] Ambrosio, L., Dal Maso, G.: On the relaxation in $B V\left(\Omega ; \mathbb{R}^{m}\right)$ of quasiconvex integrals. J. Funct. Anal. 109, 76-97 (1992)

[6] Ambrosio, L., Fusco, N., Pallara, D.: Functions of Bounded Variation And Free Discontinuity Problems. Clarendon Press, Oxford (2000)

[7] Bouchitté, G., Fonseca, I., Mascarenhas, L.: Relaxation of variational problems under trace constraints. Nonlinear Anal. 49, 221-246 (2002)

[8] Choksi, R., Fonseca, I.: Bulk and interfacial energy densities for structured deformations of continua. Arch. Rat. Mech. Anal. 138, 37-103 (1997)

[9] Dacorogna, B.: Direct Methods in the Calculus of Variations. Springer, Heidelberg (1989)

[10] Dacorogna, B., Fonseca, I., Malý, J., Trivisa, K.: Manifold constrained variational problems. Calc. Var. Partial Differ. Equ. 9, 185-206 (1999)

[11] De Giorgi, E., Ambrosio, L.: Un nuovo tipo di funzionale del calcolo delle variazioni. Atti. Accad. Naz. Lincei Rend. Cl. Sci. Fis. Mat. Natur. 82, 199-210 (1988)

[12] De Simone, A.: Energy minimizers for large ferromagnetic bodies. Arch. Rat. Mech. Anal. 125, 99-143 (1993)

[13] Del Piero, G., Owen, D.: Structured deformations of continua. Arch. Rat. Mech. Anal. 124, 99-105 (1993)

[14] Fonseca, I., Leoni, G.: Relaxation results in micromagnetics. Ricerche Mat. 49, 269-304 (2000)

[15] Gioia, G., James, R.D.: Micromagnetics of very thin films. Proc. R. Soc. Lond. Ser. A 453, 213-223 (1997) 
[16] Morrey, C.B.: Multiple Integrals in the Calculus of Variations. Springer, Heidelberg (1966)

P. M. Santos

Instituto Superior Tecnico, Av. Rovisco Pais,

1049-001 Lisbon, Portugal

e-mail: pmsantos@math.ist.utl.pt

Received: 06 November 2007.

Revised: 14 October 2008.

Accepted: 07 January 2009. 